\section{Basics of EEG Monitoring in Pediatric Critical Care}

Coral M. Stredny and Mark H. Libenson

\section{LEARNING OBJECTIVES}

- To review basic principles of EEG

- To recognize features of the normal pediatric EEG

- To understand the basic principles of referential and bipolar montages

- To identify EEG patterns specific to ICU recordings

\section{Introduction}

Since the first recording of the electroencephalogram in humans by Hans Berger nearly 100 years ago, EEG has been used in an increasingly sophisticated fashion as a noninvasive means to identify seizures, to localize and assess cerebral dysfunction, and to aid in determining neurologic prognosis. ${ }^{1}$ Over the past decade, the use of EEG in the pediatric ICU has expanded rapidly. ${ }^{2,3}$ This chapter provides an overview of the basic principles of EEG monitoring with an emphasis on ICU recordings.

In order to appreciate abnormalities that appear in the EEG tracing, it is important to be familiar with the normal patterns of infancy, childhood, and adolescence. The EEG of term and preterm neonates changes dramatically according to gestational age and is outside the scope of this discussion.

\section{Basic Principles of EEG Relevant to the ICU}

\section{Basic Neuronal and Synaptic Physiology}

The billions of neurons in the human brain communicate via electrical signaling, and it is the composite of these electrical signals that is captured on the surface EEG. It has been estimated that scalp electrodes detect the activity of an approximately $6 \mathrm{~cm}^{2}$ area of underlying cortex, with the ability of each electrode to detect electrical activity dependent on the intensity of the activity and its distance from the cortical surface. Of note, the activity generated by Copyright Springer Publishing Company. All Rights Reserved. From: Atlas of Pediatric and Neonatal ICU EEG DOI: $10.1891 / 9780826148674.0001$ cortical wave generators located deep in the brain may be indirectly measured by scalp electrodes given that they influence neurons on the cortical surface, which are preferentially recorded by scalp EEG. ${ }^{4}$

EEG discharges are either surface-negative or surface-positive in polarity, and the epileptic spike, which tends to be scalp-negative, is a useful example for understanding polarity. Consider first that $\mathrm{Na}^{+} / \mathrm{K}^{+}$pumps in the cell membrane constantly pump three $\mathrm{Na}^{+}$ions out of the cell for every two $\mathrm{K}^{+}$ ions pumped into the cell. This results in the establishment of the well-known transmembrane gradient $-\mathrm{Na}^{+}$is more plentiful outside of the cell while $\mathrm{K}^{+}$is more plentiful inside the cell. This 3-for-2 exchange of cations leaves a positive charge on the extracellular membrane and a negative charge on the intracellular membrane. Neuronal excitation is a reversal of this polarized negative intracellular and positive extracellular resting state. For example, opening of $\mathrm{Na}^{+}$or $\mathrm{Ca}^{++}$channels prompts an inflow of cations into the cell along both charge and concentration gradients, resulting in depolarization of the membrane; this can trigger an action potential if the magnitude of the depolarization is sufficient. Excitatory neurotransmitters (e.g., glutamate) function in this manner, opening channels that allow cations into the nerve cell, thereby causing the membrane to depolarize. The depolarization that these neurotransmitters create is called an excitatory postsynaptic potential (EPSP). Conversely, inhibitory neurotransmitters (e.g., gamma-aminobutyric acid [GABA]) open channels that allow $\mathrm{Cl}^{-}$ions to flow into the cell, thus increasing the baseline intracellular negativity. This causes a hyperpolarization that makes the inside of the cell membrane even more negative and is called an inhibitory postsynaptic potential (IPSP)., ${ }^{4,5}$

Epileptic spikes represent sudden, synchronous excitatory inputs on a group of pyramidal cell bodies. Up to now, we have been observing these charge shifts from perspectives both inside and outside of the neuron, but now let's consider the EEG electrode's point of view, which observes extracellular voltage changes from its perch on the scalp. As mentioned, EPSPs open cation channels and facilitate the flow of positively charged ions away from the scalp electrode-they can be thought of as disappearing into the neuron's intracellular space. This disappearance of positivity is sensed by the electrode as a negative voltage event. Indeed, the inward shift of cations is balanced by compensatory ion shifts deeper in cortex so the net charge remains more or less steady, but because these compensatory shifts occur deeper in cortex, they are not as well "seen" by the EEG electrode. For this reason, the large majority of epileptic spikes recorded at the scalp show negative polarity. ${ }^{4}$

Thus, the EEG waves we record at the scalp represent the summation of the EPSPs and IPSPs that impinge on neurons at the cortical surface. Different regulators of these cortical potentials, including thalamocortical projections, can lead to neuronal synchronization and the formation of a variety of both normal and epileptiform rhythms. ${ }^{4,5}$ 


\section{Electrode Types and Imaging Compatibility}

Most EEG electrodes are cup-shaped and are applied to the scalp using an electrolyte paste. The electrode is made of a metal that shares a common ion with the paste so as to allow the free flow of anions; most commonly, silver-coated silver chloride electrodes are used. The skin in the area of electrode placement is prepared prior to application by removing dead skin cells and oils in order to decrease electrode impedance. ${ }^{6,7}$

Critically ill patients in the ICU often require anticipated and unanticipated neuroimaging studies. Standard metal electrodes are not compatible with MRI scanners and create significant artifact when used in conjunction with CT scanning. Removal and replacement of the electrode set for imaging may be time-consuming and upsetting for the conscious patient. MRI-compatible electrodes crafted from silver, nylon, and Teflon may be used, ${ }^{8}$ with the caveat that some electrode positions (e.g., certain references, electromyography [EMG], and EKG leads) may need to be eliminated if those electrodes are not safety-approved for the MRI-compatible electrode set. Materials for such electrode systems must be chosen such that the electrode and attached wire will not heat up in the MRI's magnetic field or disturb image production. Similar plastic electrodes are also available that are CT-compatible and create minimal artifact. ${ }^{9}$

Because EEG recordings in the ICU are often prolonged, regular surveillance for skin breakdown at the electrode sites should be performed according to a set protocol. At our institution, we use the following schedule for skin checks: An EEG technologist checks scalp skin every 24 hours during continuous EEG (cEEG) monitoring, particularly under Fp1, Fp2, F7, F8, O1, and O2, the electrode positions under which skin is most susceptible to breakdown. If skin irritation is found, affected leads are moved to neighboring 10-10 locations (see below). For example, if there is irritation at Fp1 and Fp2, these leads are moved to the AF3 and AF4 positions. A new montage is created with the relocated leads.

\section{Choice of Montage}

\section{0-20 System and Types of Montages}

Electrodes are applied according to the International 10-20 system, a system for measuring and naming EEG electrode positions. ${ }^{10}$ The name "10-20" is derived from the strategy of making initial standard head measurements, then placing electrodes at intervals of $10 \%$ or $20 \%$ between those standard landmarks (Figure 1.1). ${ }^{11}$ Once 19 or more EEG electrodes have been placed according to the 10-20 system, pairings of electrodes are chosen for display, and each such pairing (e.g., Fp1-F3) is referred to as a derivation. The voltage difference between the two electrodes in each derivation is displayed on the screen as an EEG channel and constitutes the essential element of the electroencephalogram. The two electrode inputs of any channel (e.g., Fp1 and F3) are attached to the two poles of a common mode rejection amplifier (CMR). The CMR amplifier allows for the second input to be subtracted from the first input such that any noise signal common to both channels is canceled out. The difference signal (whatever is different between inputs 1 and 2) is amplified. Without use of the CMR amplifier, the environmental noise that runs through the body would swamp the low-voltage EEG signal from the brain, making the tracings uninterpretable.

The individual channels are then arranged as a montage, a specific, logical ordering of a series of electrode channels on an EEG page. Ideally, an EEG montage is constructed such that it is easy to interpret by the reader, displays EEG information accurately, and limits the amount of electrical noise or artifact that obscures the tracing. In general, it is recommended that montages be set up such that channels are displayed with left hemispheric chains presented over right hemispheric chains and anterior electrodes over posterior electrodes, using consistent interelectrode distances. ${ }^{11}$

\section{Bipolar Versus Referential Montages}

Imagine an EEG setup with five electrodes on the head, labeled A, B, C, $\mathrm{D}$, and E. In a bipolar montage, the recording from the five electrodes is displayed in four channels: A-B, B-C, C-D, and D-E. In fact, the use of these "bipolar chains" is the most common strategy for displaying EEG signals. Alternatively, in a referential montage, each of the five electrodes is compared to a location on the body that is felt to be electrically quiet, such as the chin. For example, a referential montage using the chin for these five electrode positions would be displayed in five channels as: A-chin, B-chin, C-chin, D-chin, and E-chin. Electrodes that are not involved in the field of a discharge can also be used as a referential montage. For example, the $\mathrm{Cz}$ electrode can be used as a reference, where each electrode is compared to $\mathrm{Cz}$ (e.g., $\mathrm{A}-\mathrm{Cz}$, $\mathrm{B}-\mathrm{Cz}, \mathrm{C}-\mathrm{Cz}, \mathrm{D}-\mathrm{Cz}, \mathrm{E}-\mathrm{Cz})$. A summation of several electrodes can also be used, where each electrode is compared to the average electrical charge of all of the electrodes on the head (e.g., A-Avg, B-Avg, C-Avg, etc.).

Most EEG screening is done in bipolar montages. Because the pairing of nearby electrodes in bipolar chains minimizes noise, bipolar montages tend to look "cleaner" and are easier to scan, although they do have inherent technical limitations. Localization of discharges and determination of whether a particular wave is artifact or of cerebral origin is often aided by reexamining an EEG page using a referential montage. Referential montages are more prone to electrical noise but have distinct advantages as well. In summary, a bipolar montage compares each electrode to a neighboring electrode, while a referential montage compares each electrode to a common electrode or summation of electrodes (e.g., average reference). 


\section{Bipolar Montages}

The two types of bipolar montages used most often are anteroposterior (AP), in which electrode chains are set up from the front to the back of the head (Figure 1.2), and transverse, in which electrode chains are set up from the left to the right side of the head (Figure 1.3). This allows for scanning of the voltage differences in two different orthogonal planes across the scalp. The transverse bipolar montage also allows the reader to assess abnormalities at the ends of the electrode chains (e.g., O1/O2, Fp1/Fp2) more effectively than does the AP bipolar montage, given that, in an AP bipolar montage, end-of-chain electrodes are compared to only anterior (in the case of Fp1 and Fp2) or posterior electrodes (in the case of $\mathrm{O} 1$ and O2; Figure 1.3)

Care should be used when creating montages to ensure that the interelectrode distance remains consistent in a bipolar montage whenever possible. Longer interelectrode distances create the effect of larger potential differences and apparent higher channel amplitudes (Figure 1.4), while shorter interelectrode distances create the effect of smaller potential differences and apparent lower channel amplitudes (Figure 1.5), even though the voltages generated by the underlying cortex are the same, creating a false asymmetry. If this is suspected, one should confirm the finding on a referential montage (Figure 1.5).

\section{Referential Montages}

When setting up a referential montage, how does one choose the ideal comparison point or reference electrode to compare to all the scalp positions? The ideal reference is one that "includes all of the electrical noise in the 'electrode of interest' but none of the electrocerebral activity."12 The ideal comparison electrode would cancel out all of the noise from the scalp electrode of interest but would not inadvertently subtract out any of the electrical activity from the brain. Unfortunately, such an ideal reference electrode does not exist and compromises have to be made, but a reference position far enough from the electrode of interest to not contain much of its electrocerebral activity, but close enough to cancel out the common noise signal, is the goal. Possible reference electrode locations include the spinous process of C7 (CS2), the earlobes (A1 [left] and A2 [right]), the nose, or, as noted earlier, the chin or the vertex $(\mathrm{Cz})$. Particularly in young children or those with sensory sensitivities, reference electrodes can be easily pulled off and are prone to myogenic artifact, though this may be less of a problem in ICU settings where patients are often comatose or sedated.

As noted earlier, a more complex type of reference electrode can be created using a simple arithmetic average of the electrode set, referred to as an average reference electrode. With this scheme, a voltage that is the average of all of the scalp electrodes is attached to input 2 of the CMR. This strategy has its own inherent advantages and disadvantages. Average reference montages are most useful for localizing focal, low- to medium-voltage epileptiform discharges as these do not tend to affect the average very much. Broader discharges or those with high voltages tend to "contaminate" the average reference, leading to deflections in all channels to which it is compared and risking false localization. ${ }^{12}$

\section{Special Situations/Limited Montages}

For some patients, it may be necessary to place a limited set of electrodes because some portions of the scalp may be inaccessible (due to surgical incisions or inability to turn the head). A custom montage may be created or a limited set of electrodes may be placed as a double-distance or even more limited electrode array. Limited montages also have the advantage of being simpler to apply, potentially permitting greater use of cEEG in resource-limited settings where EEG technologists may not be available or may be available in a limited capacity. The most common limited montage used in the ICU setting is the subhairline montage, in which stick-on electrodes are placed below the hairline by a bedside provider. These have been shown to have a high specificity for the detection of seizures and interictal epileptiform abnormalities; however, the sensitivity for seizure detection and epileptiform discharges is quite low. In a large study comparing full to limited-montage EEG, 170 adult ICU patients had an abbreviated montage of electrodes placed below the hairline and a full-montage 10-20 placement of electrodes as a reference. ${ }^{13}$ The 4-channel subhairline limited montage had a low sensitivity for the detection of both seizures $(0.54,95 \%$ confidence interval [CI] 0.29-0.77) and interictal epileptiform activity $(0.60,95 \%$ CI $0.46-0.74)$, although the specificity was high for seizures $(1.00,95 \%$ CI $0.97-1.00)$ and for interictal epileptiform activity (0.94,95\% CI 0.88-0.97). Similarly, a comparison of 70 patients using a 4-channel subhairline limited montage and a full-montage 10-20 placement of electrodes showed a sensitivity of $68 \%(95 \%$ CI $45-86 \%)$ and a specificity of $98 \%$ (95\% CI 89-100\%) for detection of seizures. ${ }^{14}$ Westover et al. ${ }^{15}$ hypothesized that discrepancies between limited and full montages may in part be due to inter-reader variability. It also appears that the configuration of the reduced montage impacts performance. A large study of a computational algorithm for the detection of ictal and other patterns showed a high sensitivity and specificity for a "hairline + vertex" montage and a "forehead + behind ear" montage in comparison to full electrode arrays. ${ }^{16} \mathrm{~A}$ study examining the performance of a headset-type continuous video EEG monitoring system from NihonKohden (electrodes over the left and right frontal, temporal, central, and occipital regions) in comparison to a full-montage 10-20 placement of electrodes showed a sensitivity for detecting nonconvulsive status epilepticus of 0.706 (95\% CI $0.440-0.897)$ with a specificity of 0.970 (95\% CI $0.842-0.999) .{ }^{17}$ While 
potentially useful as a screening tool in limited resource settings, reduced electrode arrays do have limitations that must be considered when using these for the management of critically ill patients in the ICU.

\section{Principles of EEG Localization}

In bipolar montages, in which successive electrodes in a chain are sequentially paired, for example, A-B, B-C, the point of maximum negativity or positivity is found by identifying a phase reversal, or the point going through the chain where the waveform flips from down to up or up to down in successive channels (Figures 1.6A, 1.7A). When the wave flips from down to up and the tips in the phase-reversing channels are nearest to each other, this represents a point of maximum surface negativity. In instances where the waves flip from up to down and the waves appear to bulge away from each other, this represents a point of maximum surface positivity. It is important to remember that the concept of the phase reversal allows localization of not just epileptiform activity, but any type of wave, including slow waves, artifacts, and so on. In other words, the presence of a phase reversal does not provide evidence that a particular discharge is an epileptiform discharge; rather it identifies the point of maximum positivity or negativity on the scalp.

The convention regarding the polarity or direction of deflection for EEG waves viewed on a referential montage is as follows: if the input 1 electrode is more negative than the input 2 electrode, the wave goes up, and if the input 1 electrode is more positive than the input 2 electrode, the wave goes down. This makes the approach to identifying the location of maximum voltage simple: the larger the wave, the greater the voltage. Assuming the ideal situation where the voltage at the referential electrode is more-or-less neutral, the largest up-going wave marks the location of the greatest negativity and, likewise, the largest down-going wave represents the location of maximum positivity. (Figures 1.6B and 1.7B). ${ }^{12}$

\section{Visual Analysis of the EEG}

Many EEG tracings in the critical care setting are abnormal and reflect varying degrees of cerebral dysfunction. It is useful to be familiar with the appearance of the normal EEG in order to appreciate the degree to which any abnormal tracing deviates from normal. In Table 1.1, commonly used EEG descriptors are summarized.4,12,18 This section will briefly review the appearance of the normal pediatric EEG focusing on normal features and variants, especially those that could be confused for an abnormal pattern in the ICU.
By 3 months of age, the normal EEG demonstrates an AP gradient during wakefulness. This term refers to the tendency of faster frequencies to be seen in the front of the head and slower frequencies in the back. The second feature of the gradient is that voltages tend to be higher in the posterior head regions and lower in the anterior regions. Thus, the AP gradient includes two separate gradients: decreasing frequency and increasing amplitude going from front to back.

The simplest expression of this idea is that we often see low-voltage fast activity in the front of the head and slower, higher-voltage activity toward the back, in the form of the posterior rhythm or posterior dominant rhythm (PDR; Figures 1.8A and 1.9). One should note that the PDR has inherent asymmetry in voltage with lower voltage over the left as compared to the right hemisphere. If the amplitude of the PDR over the right hemisphere is more than three-fold greater than the amplitude of the PDR over the left hemisphere, it is considered abnormal. In contrast, if the amplitude of the PDR is greater than two-fold higher over the left hemisphere as compared to the right hemisphere, it is considered abnormal. This applies to patients who have a dominant left hemisphere. In patients with a dominant right hemisphere, the earlier amplitude criteria are reversed. ${ }^{12}$

The tracing should otherwise be symmetric, meaning that frequency and voltages are similar in homologous brain regions. Persistent asymmetry of normal background features is suggestive of a structural or functional abnormality in the hemisphere with the poorly developed or absent normal features. Reactivity should be present in all states, with a change in the EEG appreciated in response to external stimuli. Lack of these key characteristics is suggestive of encephalopathy and/or a structural or functional abnormality in the case of asymmetry. ${ }^{4}$ Typical ages of onset of normal background features are summarized in Table 1.2.

A child should cycle through several states during a prolonged recording, including wakefulness, drowsiness or Stage 1 sleep (N1), Stage 2 sleep (N2), and deeper slow wave sleep (N3). Rapid eye movement (REM) sleep may also be seen (Figure 1.8B-H). Epileptiform discharges can be activated with transition from wakefulness into Stage 1 and Stage 2 sleep. ${ }^{4}$

\section{Wakefulness}

Wakefulness is often associated with eye blink, muscle, and motion artifact in the EEG. The PDR is the primary identifiable rhythm seen during wakefulness. It appears with eye closure and disappears when the eyes are open and there is visual fixation (Figures 1.8A and 1.9). It first appears at 3 to 4 months of age with a frequency of 3 to $4 \mathrm{~Hz}$ and progressively increases to $10 \mathrm{~Hz}$ by 15 years of age. Table 1.3 summarizes the typical frequency of the PDR by age, as well as minimum normal values for age. ${ }^{19,21}$ 
TABLE 1.1 VISUAL ANALYSIS OF PEDIATRIC EEG

\begin{tabular}{|c|c|}
\hline $\begin{array}{l}\text { Descriptive } \\
\text { Characteristic }\end{array}$ & Definition \\
\hline Frequency & $\begin{array}{l}\text { Rate at which a waveform repeats, measured in cps or } \mathrm{Hz} \text { and often described in terms of specific frequency bands: } \\
\text { Delta: } 0-<4 \mathrm{~Hz} \\
\text { Theta: } 4 \text { to }<8 \mathrm{~Hz} \\
\text { Alpha: } 8-13 \mathrm{~Hz} \\
\text { Beta: }>13 \mathrm{~Hz}-30 \mathrm{~Hz} \\
\text { Gamma: }>30 \mathrm{~Hz}\end{array}$ \\
\hline Voltage & $\begin{array}{l}\text { Difference in amplitude between two points in a single channel derivation (e.g., Fp1-F7), calculated by multiplying the height of the wave on the page } \\
(\mathrm{mm}) \text { by the sensitivity }(\mu \mathrm{V} / \mathrm{mm}) \text { or using digital measurement tools }\end{array}$ \\
\hline Duration & Time between waveforms or time from start to end of a single waveform \\
\hline Polarity & Describes positivity or negativity of an electrical event on the scalp \\
\hline Morphology & Shape of waveform (e.g., spike, sharp wave, sharply contoured, sinusoidal) \\
\hline Location/Distribution & $\begin{array}{l}\text { Description of which electrodes are involved in a waveform: focal (single or other highly restricted location), regional (multiple electrodes covering a } \\
\text { portion of, but not the entirety of, a hemisphere), hemispheric (involving the entirety of the left or right hemisphere), generalized (entire cortex/scalp), or } \\
\text { multifocal (three or more locations involving both hemispheres) }\end{array}$ \\
\hline State & Awake, drowsy, or asleep \\
\hline Symmetry & Similarity of waveforms in terms of amplitude, morphology, and frequency, comparing homologous regions of the left and right hemispheres \\
\hline Rhythmicity & Measure of a wave's regularity and predictability (e.g., rhythmic, semirhythmic) \\
\hline Synchrony & $\begin{array}{l}\text { Timing of waveforms in relation to one another, usually in reference to a comparison of the right and left hemisphere; waveforms may be synchronous } \\
\text { (occurring simultaneously) or asynchronous (occurring 1.5-2 seconds apart) }\end{array}$ \\
\hline Continuity & Describes waveforms that are uninterrupted by periods of voltage attenuation or suppression \\
\hline Discontinuity & Describes waveforms that are interrupted by periods of voltage attenuation or suppression \\
\hline Reactivity & $\begin{array}{l}\text { A change in EEG pattern from an external stimulus; this may take a variety of forms, such as a transient change in the frequency or amplitude of the } \\
\text { tracing }\end{array}$ \\
\hline
\end{tabular}

cps, cycles per second; Hz, Hertz. 
TABLE 1.2 TIMELINE OF NORMAL EEG FEATURES

\begin{tabular}{|l|l|}
\hline EEG Feature & \multicolumn{1}{|c|}{ Age of onset } \\
\hline Sleep Spindles & $\begin{array}{l}1-3 \text { months (asynchronous until 18-24 } \\
\text { months) }\end{array}$ \\
\hline Vertex Waves & $\begin{array}{l}2-6 \text { months (may have an asymmetric field in } \\
\text { late childhood) }\end{array}$ \\
\hline K-Complexes & $3-6$ months \\
\hline Hypnagogic/Hypnopompic Hypersynchrony & $3-8$ months \\
\hline Posterior Dominant Rhythm & $3-4$ months \\
\hline Anterior-Posterior Gradient & 3 months \\
\hline
\end{tabular}

Sources: Ebersole J, Husain A, Nordli D Jr, eds. Current Practice of Clinical Electroencephalography. 4th ed. Wolters Kluwer; 2014.; Berry RB, Albertario CL, Harding SM, et al. for the American Academy of Sleep Medicine. The AASM Manua for the Scoring of Sleep and Associated Events: Rules, Terminology and Technical Specifications. Darien, IL: American Academy of Sleep Medicine; 2018. Version 2.5.

\section{TABLE 1.3 FREQUENCY OF THE PDR BY AGE}

\begin{tabular}{|l|l|l|}
\hline Age & Typical Frequency $(\mathrm{Hz})$ & Minimum of Normal Frequency $(\mathrm{Hz})$ \\
\hline 4 Months & $3-4$ & \\
\hline 5 Months & 5 & 5 \\
\hline 1 Year & 6 & \\
\hline 2 Years & 7 & 6 \\
\hline 3 Years & 8 & 7 \\
\hline 6 Years & 9 & 8 \\
\hline 8 Years & & \\
\hline 13 Years & 10 & \\
\hline
\end{tabular}

PDR, posterior dominant rhythm.

Source: Libenson M. Practical Approach to Electroencephalography. 1st ed. Saunders; 2010.

\section{Drowsiness/Stage 1 Sleep (N1)}

In drowsiness, the PDR slows and then eventually drops out to be replaced by prominent theta rhythms (Figure 1.8B). Artifacts related to slow roving eye movements are often seen in the frontal leads and represent another hallmark of drowsiness (Figure 1.8C). Bursts of intermittent slowing can be seen, referred to as paroxysmal drowsy slowing. ${ }^{22,23}$ In younger children, these bursts may consist of high-amplitude synchronous slowing, termed hypnagogic hypersynchrony (Figure 1.10).

By 2 to 6 months of age, Stage 1 sleep (N1) is marked by the onset of vertex waves of sleep (Figure 1.8B-C). Vertex waves (or v-waves) are sharp transients with maximal negativity over the vertex $(\mathrm{Cz})$ and a field including the adjacent $\mathrm{C} 3$ and $\mathrm{C} 4$ electrodes. In young children, vertex waves may be especially sharp in contour and may be mistaken for epileptiform activity ${ }^{12}$ (Figure 1.11A-B). Of note, vertex waves in children may also be anteriorly displaced (F3, Fz, F4). Any given vertex wave or run of vertex waves may be asymmetric, with higher amplitudes over one as compared to the other hemisphere; however, this asymmetry should not be persistent, such that vertex waves are seen elsewhere in the recording that are symmetric and/or of higher amplitude over the opposite hemisphere.

\section{Stage 2 Sleep (N2)}

Sleep spindles and K-complexes are the hallmarks of Stage 2 sleep (N2; Figure 1.8D-E, 1.11C-D). In young children, these occur in long, sinusoidal, or comb-like runs at a frequency of 12 to $14 \mathrm{~Hz}$ over the central areas bilaterally. In infants younger than 6 months of age, spindles can last for up to 10 seconds (Figure 1.11C). As patients age, spindles become shorter in duration and by adulthood, last for approximately 0.5 to 2 seconds. Prior to 2 years of age, spindles may occur independently over the right and left hemispheres (asynchronously), although the total number of spindles on each side should be roughly equivalent (Figure 1.11D). Asynchrony is defined as a burst of morphologically similar EEG activity in homologous head regions separated by more than 1.5 to 2 seconds. ${ }^{4}$ After 2 years of age, spindles should occur simultaneously over both hemispheres ${ }^{12}$ (Figure 1.8D). K-complexes are characterized by diffuse, chaotic slow wave bursts, sometimes with an intermixed spindle. They may be evoked by auditory stimuli ${ }^{24,25}$ (Figure 1.8E).

\section{Stage (N3)}

What was previously referred to as Stage 3 sleep (at least 20\% of the recording occupied by delta waves) and Stage 4 sleep (at least $50 \%$ of the recording 
occupied by delta waves) has been replaced by the single designation of N3 sleep. N3 sleep or slow wave sleep is defined as a minimum of $20 \%$ delta activity (Figure 1.8F). In children and adults with normal sleep, slow wave sleep tends to occupy the first third of the night, while the latter parts of the night are dominated by REM sleep. ${ }^{2}$

\section{REM Sleep}

REM sleep emerges after early infancy and is characterized by muscle atonia (presumably to protect against the acting out of dreams), irregular respirations, and REMs. Electrographically, after 5 months of age, sawtooth waves may be seen during REM sleep as 2 to $5 \mathrm{~Hz}$ negative sharp transients in the frontocentral areas..$^{4,19}$ REMs are visualized on the EEG as large eye-movement deflections, often with superimposed lateral rectus spikes, best appreciated in the frontal leads or by dedicated eye movement electrodes ${ }^{19}$ (Figure 1.8G)

\section{Arousal}

Arousal may be relatively uneventful, marked simply by a return of the PDR, especially in adolescents (Figure 1.8H). In infants and young children, hypnopompic hypersynchrony can be seen: dramatic high-amplitude, diffuse rhythmic slow waves upon awakening (Figure 1.12). These begin abruptly and can have an anterior, temporal, or posterior predominance. They may occur continuously or intermittently for several minutes. These are less commonly seen in older children and are rare by 12 years of age. They should not be confused with abnormal slow wave activity or an ictal phenomenon. ${ }^{26}$

Other forms of arousals that can be confused for ictal phenomena include those that manifest as a transient slow wave followed by faster frequencies that are diffuse with central or frontal predominance and then by rhythmic theta to delta activity. ${ }^{2}$ Arousal patterns with a simple electrodecrement have also been described.$^{27}$ Arousals can be precipitated by either internal or external stimuli (Figure 1.13).

\section{EEG in Encephalopathy and Coma}

EEG can be a uniquely useful tool to assess the degree of encephalopathy and coma, as well as nonconvulsive seizure activity, in the ICU, a setting in which assessment by physical examination and other modalities may be limited. While a single routine EEG can offer a snapshot of a patient's level of encephalopathy, serial EEGs or cEEG monitoring can provide additional information regarding trends toward improvement or worsening of the patient's state. The presence of abnormal slowing, voltage attenuation, or lack of reactivity provides evidence of the degree of encephalopathy, as summarized below. Other patterns that can be seen in the setting of coma and encephalopathy are described, such as rhythmic delta activity, periodic discharges, spindle coma, alpha coma, and burst-suppression patterns. Occasionally specific electrographic patterns may suggest a specific diagnosis, such as rhythmic high-amplitude delta with superimposed spikes and polyspikes (RHADS) in POLG-associated disease or lateralized periodic discharges in herpes simplex encephalitis. Specific patterns seen in coma are further discussed in Chapter 2.

\section{Attenuation of Fast Activity, Slow Waves, and Voltage Suppression}

Slow waves seen outside of slow wave sleep are generally considered abnormal. There tends to be a linear relationship between slow wave frequency and degree of encephalopathy, with slower frequencies associated with worsening encephalopathy. The relationship between the degree of encephalopathy and slow wave amplitude is more complicated. In the initial stages of encephalopathy, increasing slow wave amplitudes may be seen. Eventually, as the encephalopathy worsens, overall EEG amplitudes may decrease. Thus, decreasing voltages may be a sign of a trend toward either normalization or worsening encephalopathy. This is usually not difficult to determine when the clinical context and other EEG features, as well as the findings of prior EEGs, are taken into account. ${ }^{12}$

In mild stages of encephalopathy, intermittent slow waves may become intermixed with normal background activity. With progression of the encephalopathy, the slow waves may become continuous and replace normal activity, with increasing amplitude and decreasing frequency (Figure 1.14). Eventually, in states of severe encephalopathy, voltage attenuation or suppression will be seen. Recordings with voltages $<20 \mu \mathrm{V}$ are considered low voltage, while those with voltages $<10 \mu \mathrm{V}$ are considered suppressed. ${ }^{28}$ While it is uncommon to capture all of these EEG stages in a single patient, it is useful to be acquainted with this progression in order to be able to compare various degrees of abnormality.

In a patient who progresses to brain death, no demonstrable EEG activity is present, a state which is referred to as electrocerebral inactivity (ECI). In most jurisdictions, EEG is not among the primary tests used for the legal determination of brain death, but may be used as an ancillary test to assist in the diagnosis of brain death when components of the examination or apnea testing cannot be completed safely. It can also be used to reduce the inter-examination 
period of observation. In such cases, in order to be considered a valid test, an ECI recording must fulfill certain technical criteria to ensure that the apparent lack of brain wave activity is genuine, including use of double-distance electrode linkages, sensitivities of $2 \mu \mathrm{V} / \mathrm{mm}$ for at least 30 minutes of recording, bandpass filter settings of 1 to $30 \mathrm{~Hz}$, electrode impedances of 100 to 10,000 Ohms, tapping electrodes to demonstrate continuity of the EEG circuit, and demonstrating a lack of patient reactivity to various stimuli. ${ }^{29,30}$

\section{Reactivity}

The presence of EEG reactivity is considered a positive prognostic sign. In the ICU, auditory (e.g., clapping or shouting in each ear) or tactile (e.g., stimulating each extremity) stimuli are useful maneuvers to assess reactivity. In a patient with preserved reactivity, this results in a change in EEG amplitude, rhythmicity, and/or frequency (Figure 1.15). The American Clinical Neurophysiology Society recommends that testing for EEG reactivity should follow a protocol and that neurodiagnostic technologists should perform bedside testing for reactivity at least once a day. ${ }^{31}$ It is recommended that visual, auditory, tactile, and painful stimulation be performed. ${ }^{31}$ At our center, EEG technologists perform auditory stimulation (clapping), tactile stimulation (light touch of each extremity), and noxious stimulation (nail bed pressure). While this is performed at the start of each recording, we often also rely on bedside cares to assess reactivity.

\section{Specific Patterns in Encephalopathy and Coma}

\section{RHYTHMIC DELTA ACTIVITY}

Rhythmic delta activity (RDA) may be intermittent initially but may become more continuous as the encephalopathy worsens. RDA is defined as lacking an interval between consecutive waveforms with $<50 \%$ variation in periodicity from one cycle to the next. ${ }^{28}$ RDA can be generalized (GRDA; (Figure 1.16) or lateralized (LRDA; Figure 1.17A-B) and when generalized, may demonstrate predominance either frontally, occipitally, or in the midline. In the case of GRDA, this should not be construed to localize an abnormality but rather indicates dysfunction that is not specifically localized to the region where the slowing is seen maximally. ${ }^{12}$

\section{PERIODIC DISCHARGES}

Periodic discharges (PDs) consist of regularly recurring waveforms separated by a clear interdischarge interval. They may be generalized, lateralized, bilateral independent, or multifocal. ${ }^{28}$ Lateralized PDs are often seen after an acute structural insult, such as a stroke, whereas generalized PDs tend to occur in the setting of more diffuse dysfunction. Indeed, certain patterns are quite characteristic of specific disorders in the correct clinical context, such as lateralized periodic discharges (LPDs, previously called "PLEDS"—periodic lateralized epileptiform discharges) in the temporal region in herpes simplex encephalitis (Figure 1.18). ${ }^{32}$ PDs are usually considered an interictal phenomenon, though in certain instances it may be difficult to ascertain whether PDs represent an ictal phenomenon or not (see Chapter 9). Occasionally, PDs are associated with a clear clinical correlate such as a myoclonic jerk. In most cases, attempts at treating PDs with parenteral antiseizure medications are not fruitful. ${ }^{5}$

\section{SPINDLE AND ALPHA COMA}

Spindle coma is seen in patients with a generalized slow wave pattern on EEG with embedded spindles; however, the amount of spindles is exaggerated compared to normal sleep states. Alpha coma consists of diffuse alpha frequency activity, often with frontal predominance and an absence of reactivity. While the prognosis is most dependent upon the underlying etiology of the coma, spindle coma tends to portend a better prognosis than alpha coma. Alpha coma was initially described in patients with severe brainstem dysfunction or anoxia, and in these settings, tends to carry a poor prognosis; however, when the etiology is reversible, such as drug overdose, the prognosis is less ominous. ${ }^{5,12}$

\section{BURST-SUPPRESSION}

Burst-suppression is marked by bursts of activity with intervening periods of voltage suppression and is considered an ominous pattern when seen in the setting of irreversible central nervous system injury (Figure 1.19). Bursts typically consist of polymorphic generalized spikes or polyspikes with admixed slow waves. They last for 0.5 to 30 seconds and are separated by periods of diffuse suppression that are $<10 \mathrm{uV}$ in amplitude and comprise $>50 \%$ of the recording. ${ }^{28}$ Occasionally, the bursts do not contain frankly epileptiform (sharp) activity. There is absence of variability and reactivity in the burst-suppression EEG, which is to say that the burst-suppression pattern does not come and go, but continues persistently, despite patient stimulation. As the depth of coma progresses, the bursts may become lower in amplitude and may be separated by longer interburst intervals. In patients with anoxic brain injury, burst-suppression carries a poor prognosis; however, this pattern can also be induced by pharmacologic agents, including drugs of overdose and anesthetic infusions (e.g., pentobarbital infusions), and, therefore, may be 
reversible in some cases. ${ }^{12,33}$ Patients who cycle out of a burst-suppression pattern over hours or days have a better prognosis than those patients in whom the pattern persists over several days.

\section{Localization in Encephalopathy}

Asymmetry of EEG patterns may be a clue to the presence of focal or regional central nervous system pathology (Figures 1.20 and 1.21). For example, attenuation of faster frequencies in one area may be an early localizing sign of focal cortical dysfunction and may be followed by asymmetries in slow wave amplitude and frequency (Figure 1.20). Sleep features or other normal background features may be absent over the region of dysfunction (Figure 1.21). However, it should be noted that even diffuse processes may cause EEG asymmetries in a patient with preexisting cerebral abnormalities. ${ }^{12}$

\section{EEG Review and Reporting in the ICU}

The ACNS recommends that the first 30 to 60 minutes of a cEEG be reviewed and relayed to the clinical team. Thereafter, a cEEG should be reviewed by an electroencephalographer a minimum of twice daily with more frequent interpretation based on the clinical state of the patient. Important EEG changes should be relayed to the clinical team. ${ }^{31}$

The basic principles of the EEG report hold for studies performed in the intensive care unit. Each report should include a history, including the reason for monitoring, body, interpretation, and clinical correlation. The body of the report should include a description of the EEG background, including the presence or absence of reactivity and periodic and rhythmic patterns. If quantitative EEG is used, a description of the trends analyzed should be included. A recent study describing EEG reporting for refractory status epilepticus showed significant variability in the features reported, including reactivity and seizure descriptors. ${ }^{34}$ We have included an ICU EEG report in the appendix that focuses on critical care EEG terminology.

\section{Conclusion}

Interpretation of EEG in the ICU relies on a basic understanding of the normal awake and asleep EEG. It is also important to be familiar with principles specific to pediatrics such as variation in the frequency of the posterior dominant rhythm as a function of age. Recognition of specific EEG patterns and features (e.g., alpha coma, reactivity) helps the provider gain insight into the degree of encephalopathy and can be a useful tool to aid in prognostication.
This chapter includes access to a video, Video 1.1 (Arousal), with real time EEG that illustrates concepts and patterns. Please follow the following url and click onto the show chapter supplementary tab to access the video: http:/ / connect.springerpub.com/content/book/978-0-8261-4867-4/ chapter/ch01

\section{KEY POINTS}

- EEG electrodes are applied according to the International 10-20 system and displayed in either a bipolar or referential montage.

- A bipolar montage compares each electrode to a neighboring electrode, and the point of maximum negativity appears as a negative phase reversal.

- A referential montage compares each electrode to a common electrode, which may take the form of an individual scalp electrode, an electrode placed on the ears, chin, or nose, or an average of the activity of all the electrodes. The point of maximum negativity appears as the waveform with highest amplitude.

- The normal EEG cycles through wakefulness and sleep, including drowsiness/stage 1 sleep (N1), stage 2 sleep (N2), slow wave sleep (N3), and REM sleep.

- cEEG is important to assess the degree of encephalopathy and coma, detect nonconvulsive seizures, and assess the likelihood of cerebral injury when the neurologic examination is limited.

Figures and legends for Chapter 1 begin on the next page. 


\section{Figures}

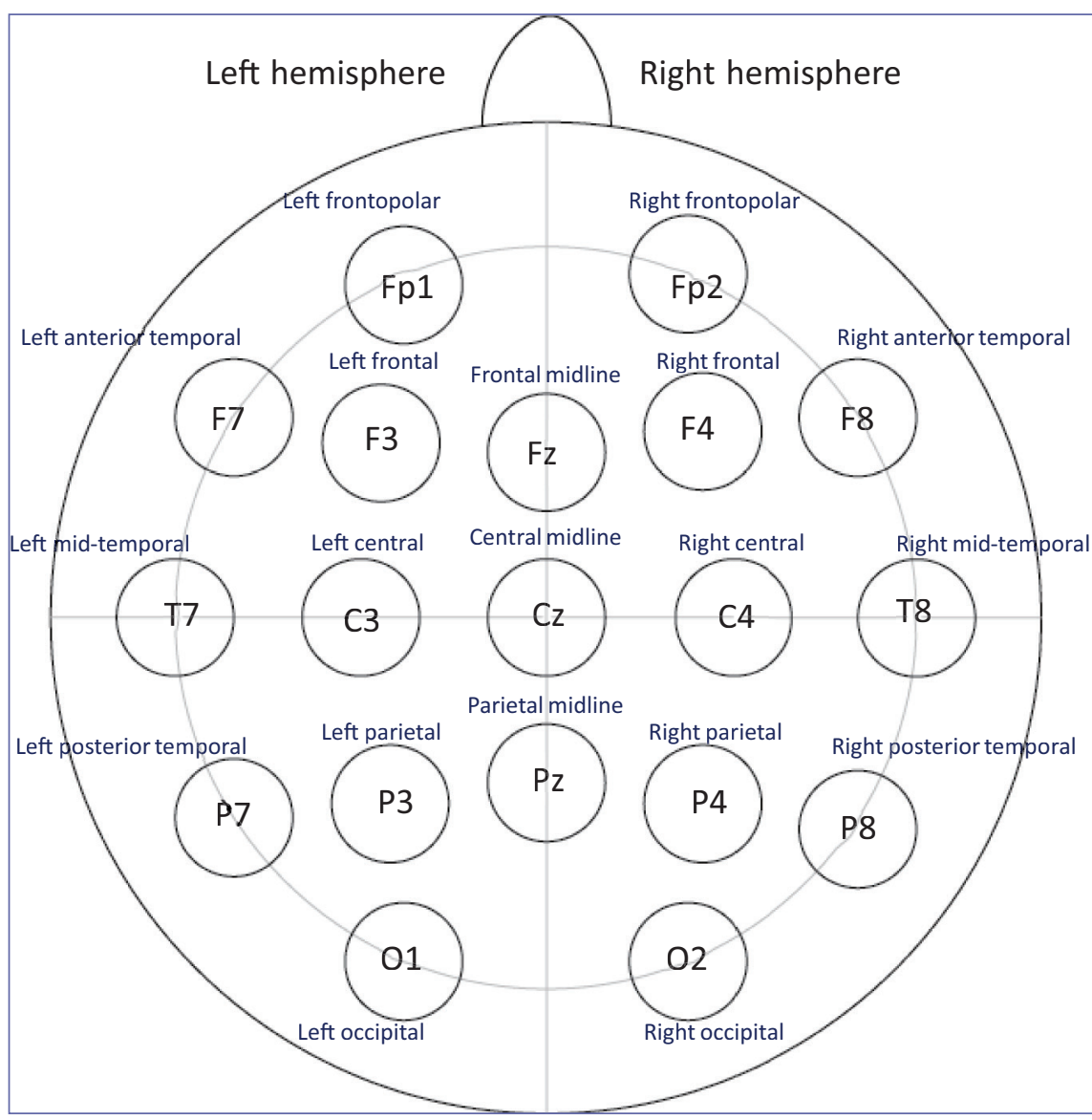

FIGURE 1.1. Electrode locations in the modified 10-20 system. Many institutions continue to use the original 10-20 nomenclature. In the original system, electrode positions T7 and T8 are referred to as T3 and T4, respectively, and positions $\mathrm{P} 7$ and $\mathrm{P} 8$ are referred to as $\mathrm{T} 5$ and $\mathrm{T} 6$, respectively. $\mathrm{Fp}=$ frontopolar, $\mathrm{F}=$ frontal, $\mathrm{T}=$ temporal,

$\mathrm{C}=$ central, $\mathrm{P}=$ parietal, $\mathrm{O}=$ occipital, $\mathrm{z}=$ midline. Note that electrodes $\mathrm{F} 7$ and F8 do not record activity over the

frontal lobe, but rather the left and right anterior temporal lobe, respectively. Similarly, the P7 and P8 electrodes do not record activity over the parietal lobe, but rather the left and right posterior temporal lobe, respectively. 

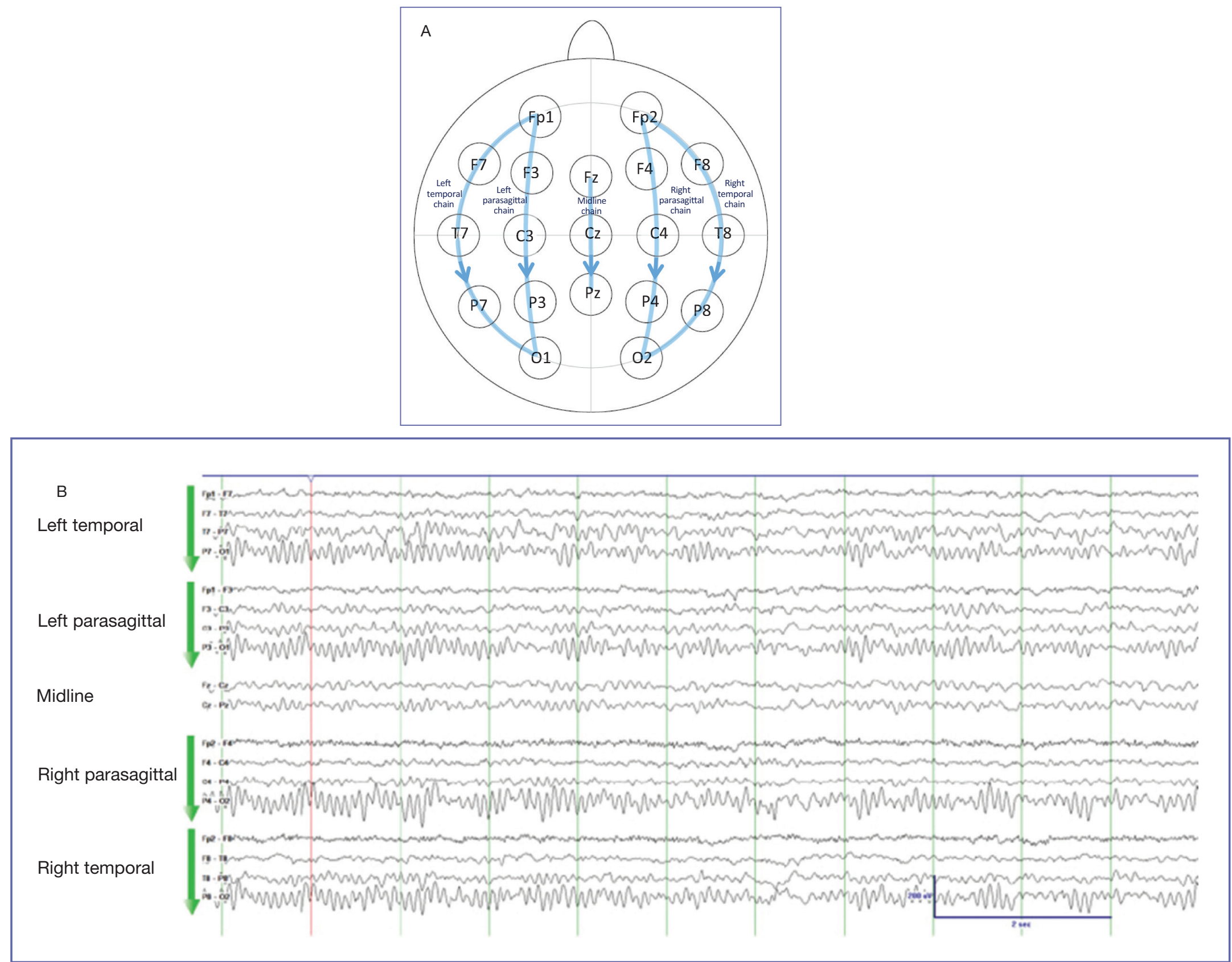

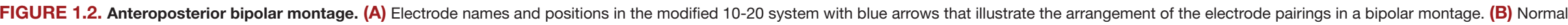

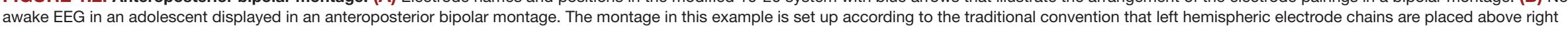

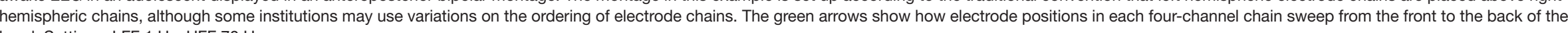
head. Settings: LFF $1 \mathrm{~Hz}, \mathrm{HFF} 70 \mathrm{~Hz}$. 
12 | Atlas of Pediatric and Neonatal ICU EEG

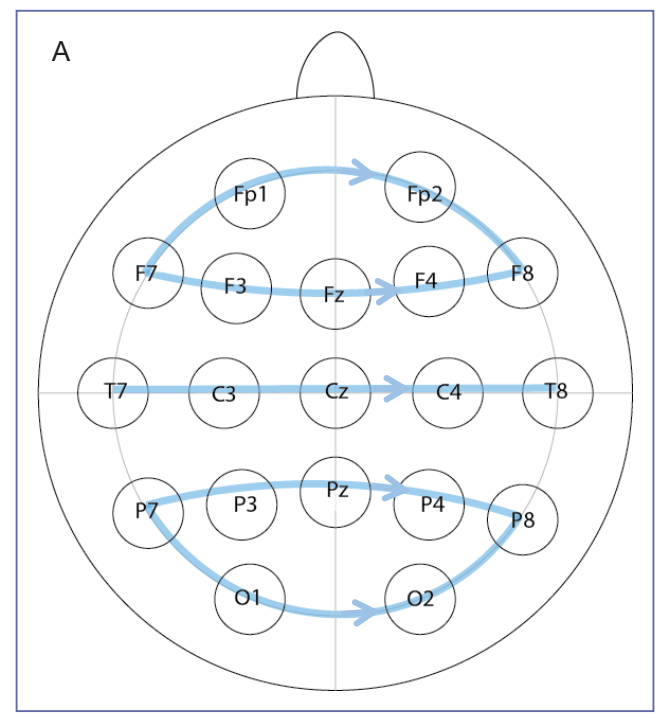

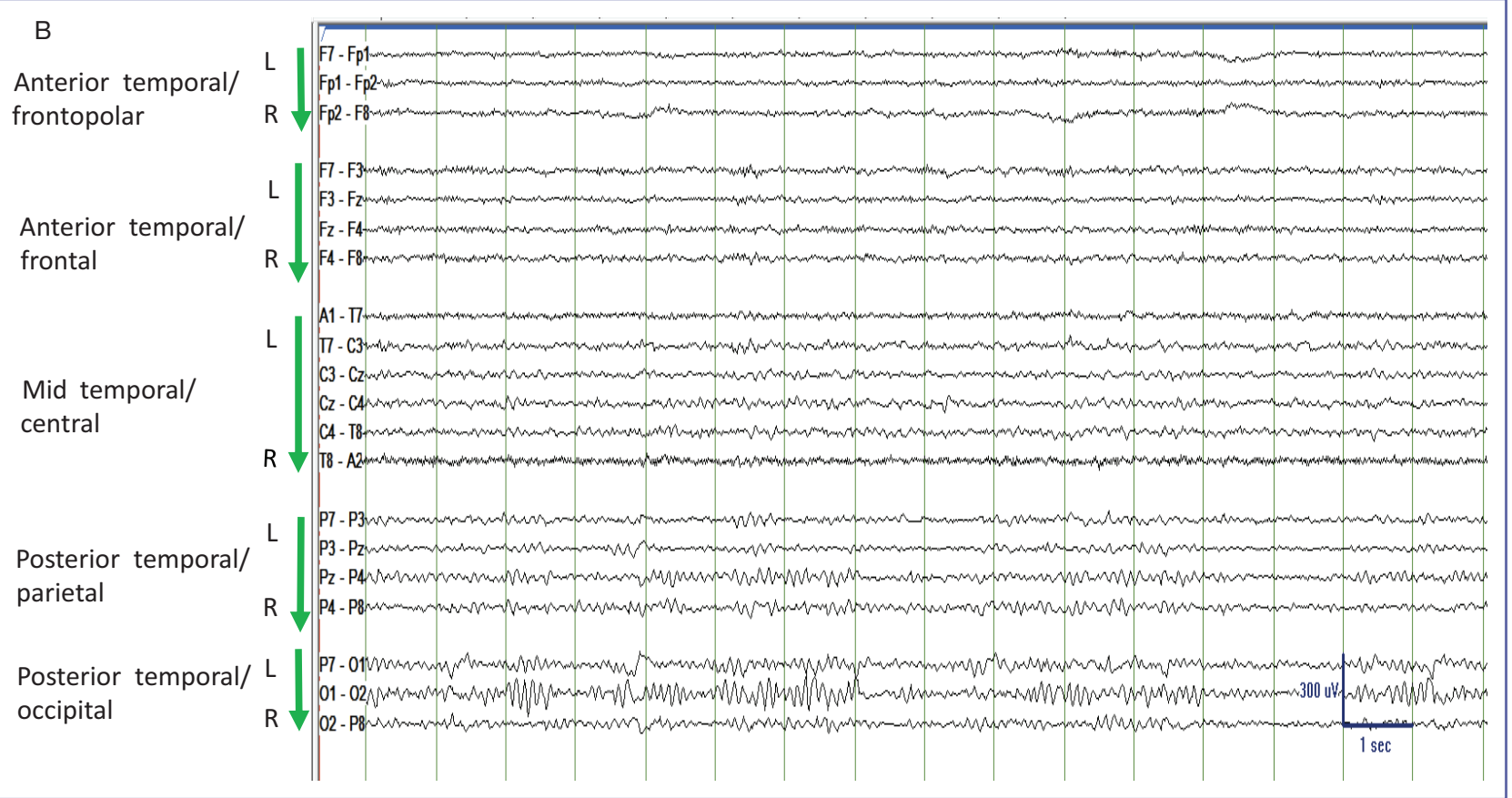

FIGURE 1.3. Transverse bipolar montage. (A) Electrode names and positions in the modified 10-20 system with blue arrows that illustrate the arrangement of the electrode pairings in a transverse bipolar montage.

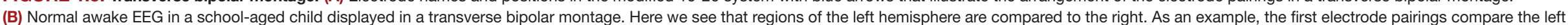
anterior temporal region and left frontopolar region to the right anterior temporal region and right frontopolar region. Subsequent regions are compared in this manner moving from the front to the back of the head. Settings: LFF $1 \mathrm{~Hz}$, HFF $70 \mathrm{~Hz}$. 


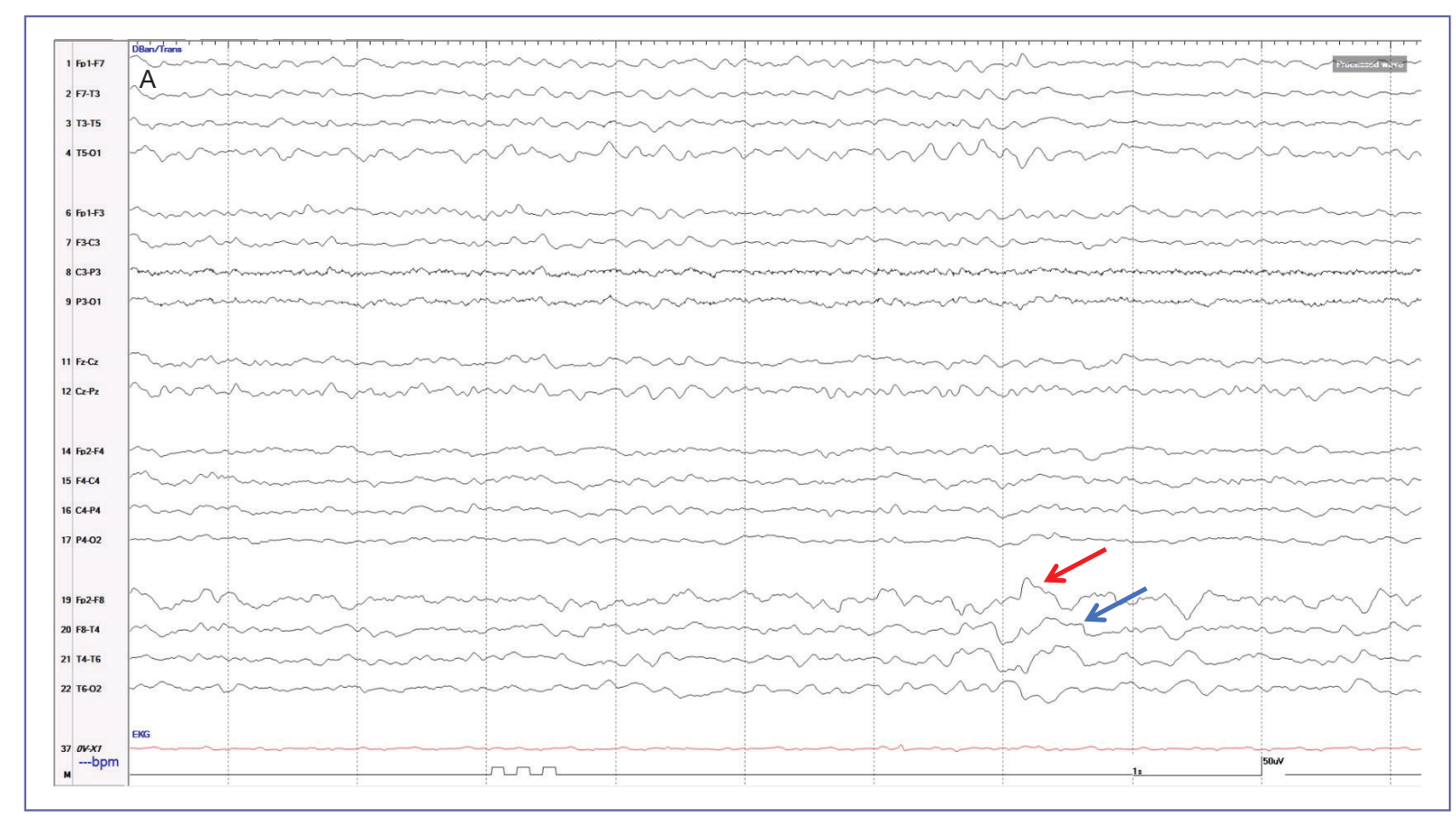

FIGURE 1.4. Effect of interelectrode distance on amplitude. A

19-month-old female with myocarditis on extracorporeal membrane oxygenation (ECMO). (A) Bipolar montage. Note the slow wave over the right frontopolar and temporal (Fp2/F8/T4[T8]) region. Using a full 10-20 system of electrodes, this slow wave measures $84 \mathrm{uV}$ in amplitude at Fp2F8 (red arrow) and 69 uV at F8-T4(T8; blue arrow). Settings: LFF $1 \mathrm{~Hz}$, HFF $70 \mathrm{~Hz}$. (B) Double distance bipolar montage. The same EEG as in Figure $1.4 \mathrm{~A}$ is shown on a double distance montage with longer inter 1.4A is shown on a double distance montage with longer interelectrode distances. Note that the slow wave over the right frontopolar and tempor (Fp2/T4[T8]) region (red arrow) is higher in amplitude, measuring $98 \mathrm{uV}$, given the longer interelectrode distance. Settings: LFF $1 \mathrm{~Hz}, \mathrm{HFF} 70 \mathrm{~Hz}$.

Source: Images courtesy of Dana Harrar.

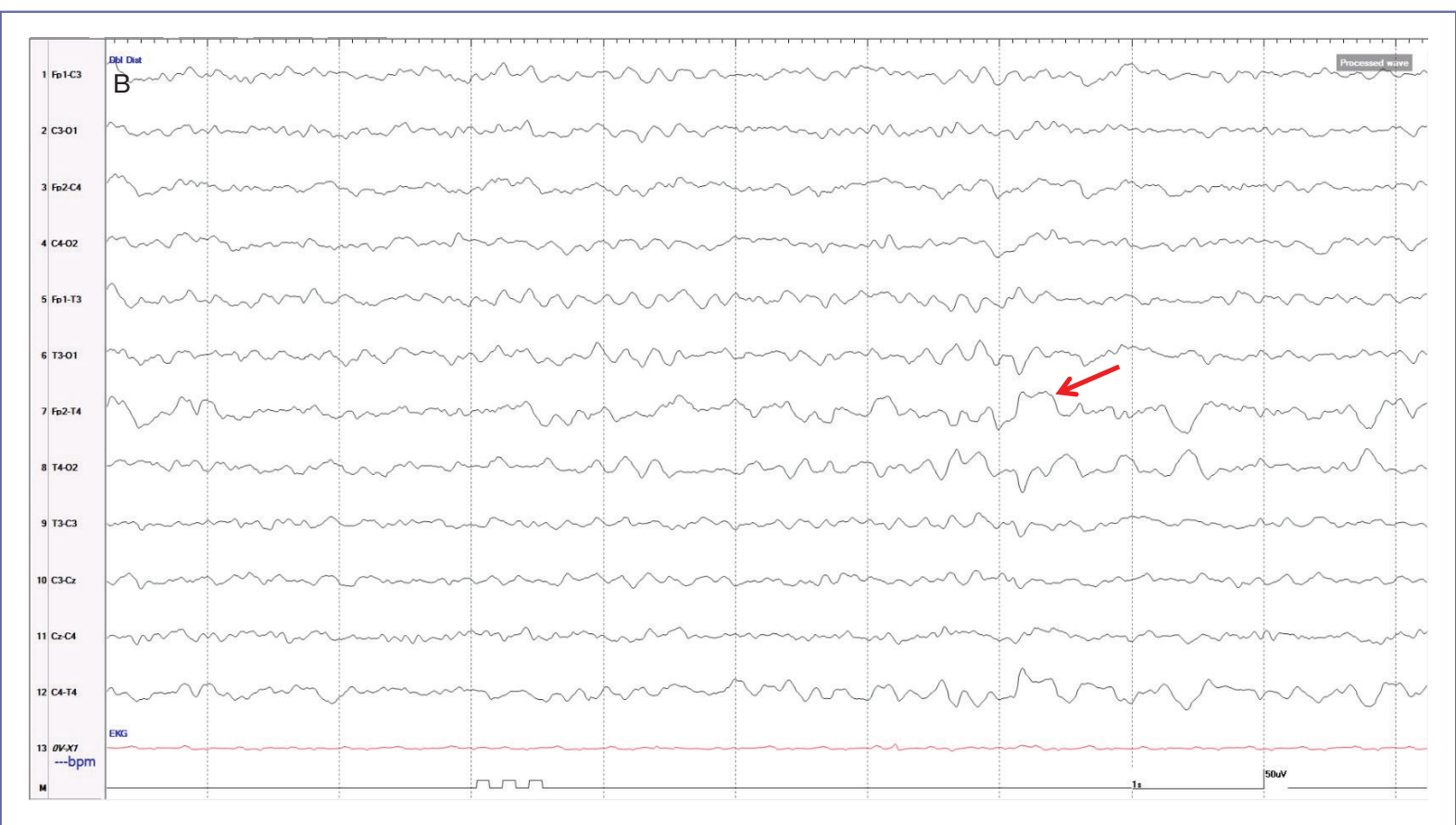



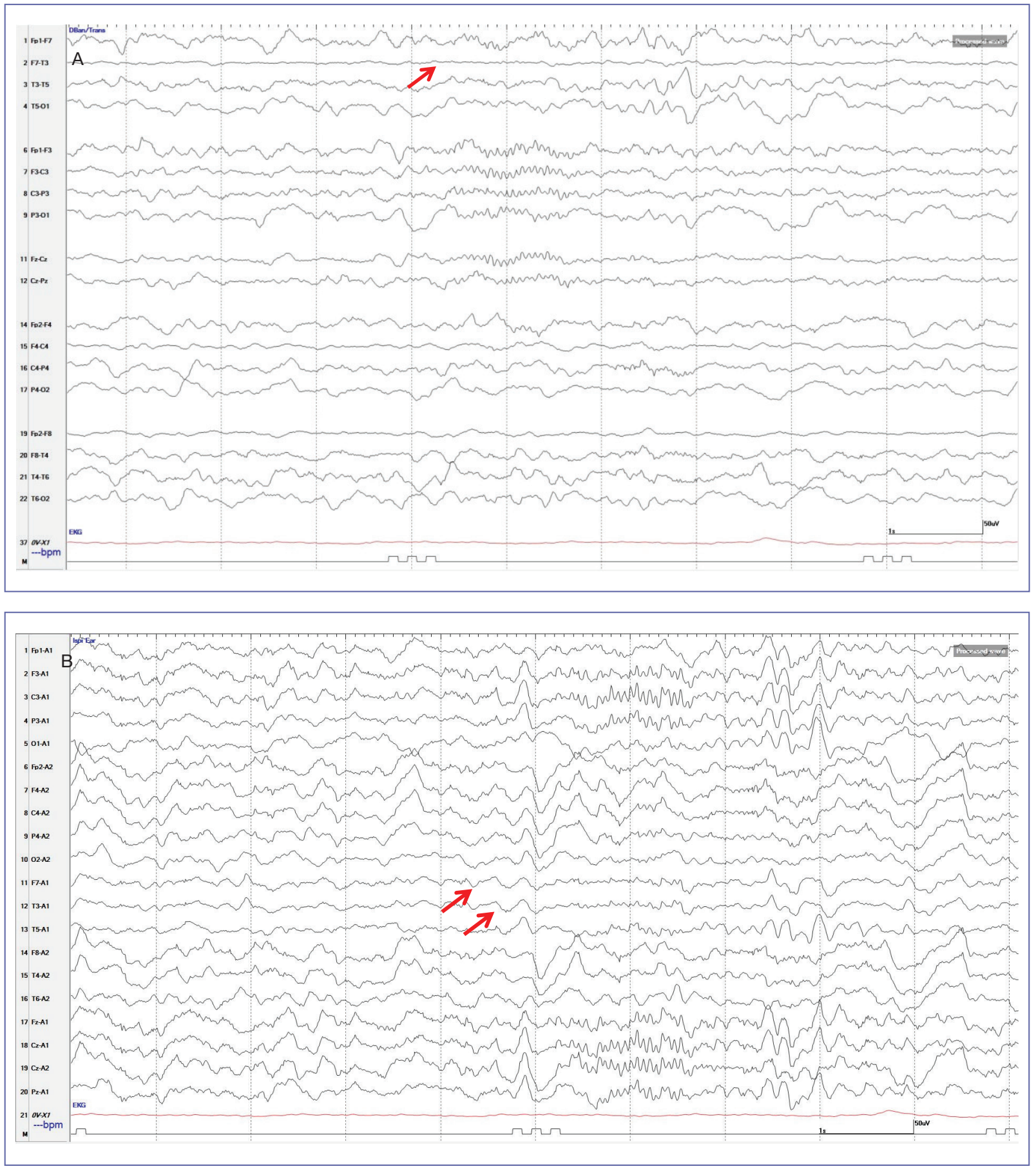

FIGURE 1.5. Effect of interelectrode distance on amplitude. A 2-year-old female with a right-sided subdural hematoma and a right>left watershed pattern of ischemic injury. (A) Bipolar montage. Note apparent voltage suppression at F7/T3(T7) due to the electrodes being placed too close together. Settings: LFF $1 \mathrm{~Hz}, \mathrm{HFF} 70 \mathrm{~Hz}$.

(B) Ipsilateral ear referential montage. The same EEG as in Figure 1.5A shown on an ipsilateral ear referential montage. Note the absence of voltage suppression at $F 7$ and T3(T7), supporting that the finding was voltage suppression at $F 7$ and T3(T7), supporting that the finding was due to lead placement rather than suppression at F7/T3(T7). Note also
that F7 and T3(T7) contain similar activity, supporting that they are recording in very close proximity to one another. Settings: LFF $1 \mathrm{~Hz}$, HFF $70 \mathrm{~Hz}$.

Source: Images courtesy of Dana Harrar. 


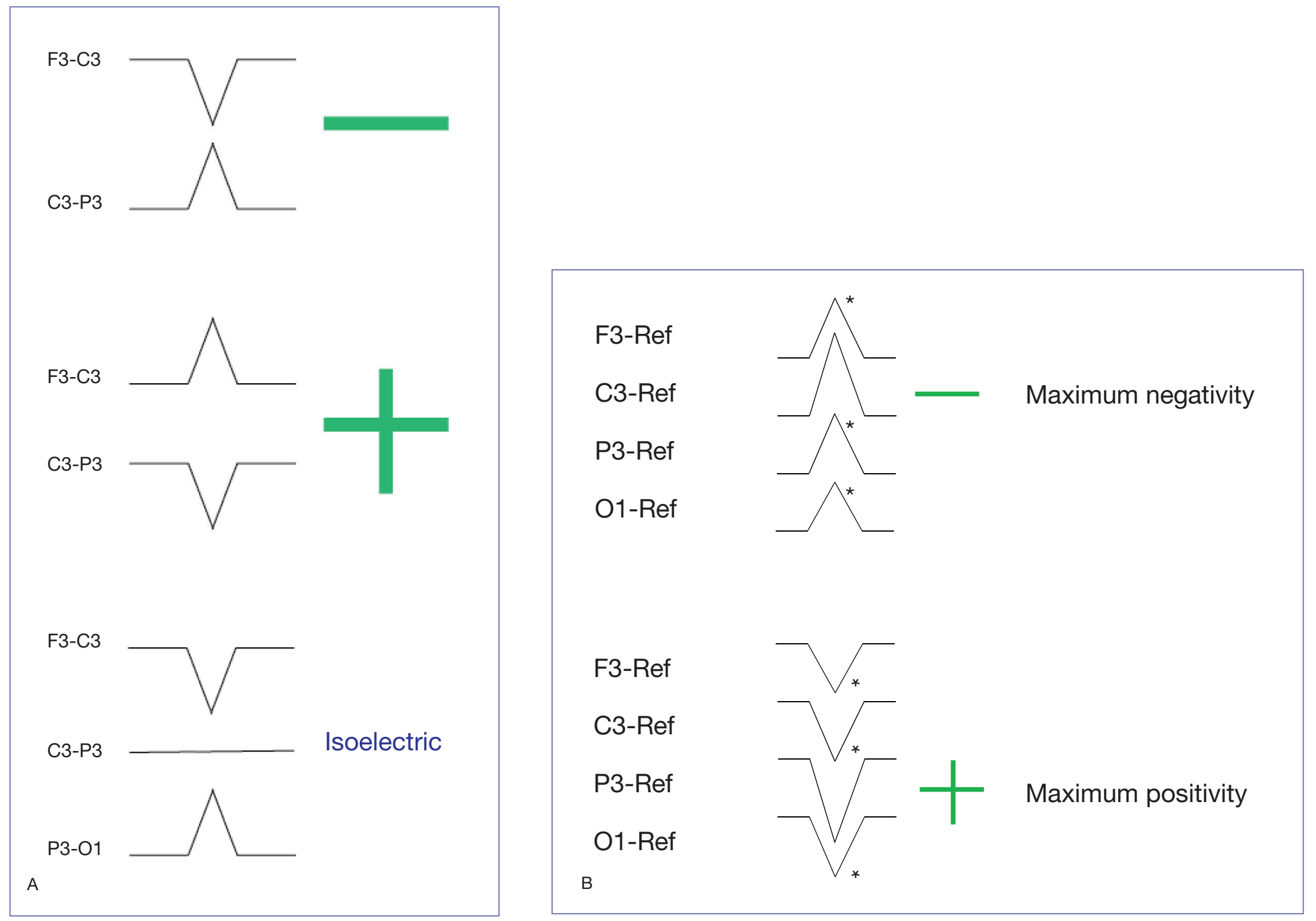

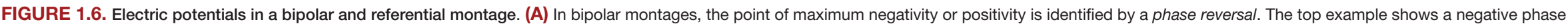

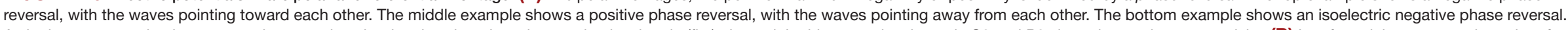

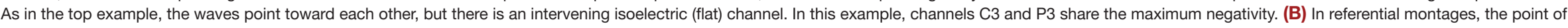

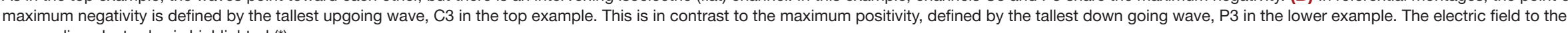
surrounding electrodes is highlighted $\left({ }^{*}\right)$. 

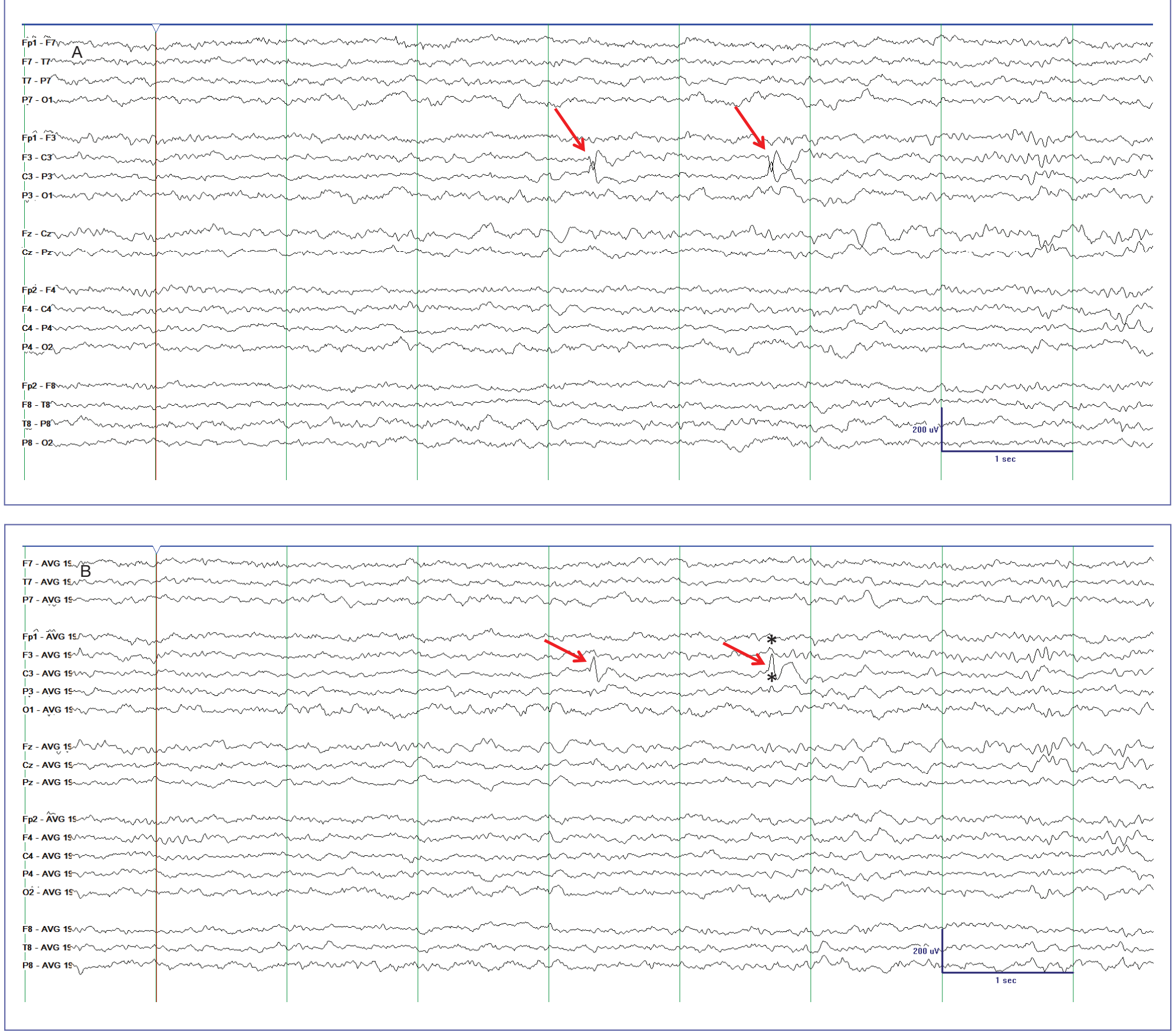

FIGURE 1.7. Bipolar and referential electric field. (A) Bipolar montage. A pair of left central (C3) spikes are shown. On an anteroposterior (AP) bipolar montage, negative phase reversals are seen at C3 (red arrows). Settings: LFF $1 \mathrm{~Hz}, \mathrm{HFF} 70 \mathrm{~Hz}$. (B) Average referential montage. The same EEG as in Figure 1.7A displayed on an average referential montage. When the same spike is displayed on an average referential montage, upward deflections with maximal negativity at $\mathrm{C} 3$ are seen (red arrows). Note that the negative spike at C3 has a small flanking electric field - there are very small upward deflections at $\mathrm{F} 3$ and $\mathrm{P3}$, upward deflections at $F 3$ and $P 3$, discharge $\left(^{\star}\right)$. Settings: LFF $1 \mathrm{~Hz}$, HFF $70 \mathrm{~Hz}$. 
FIGURE 1.8. Normal awake and asleep EEG.

(A) Bipolar montage. In this 13-year-old, the eyes are closed and a $10-\mathrm{Hz}$ posterior dominant rhythm (PDR) is present; a 1-second segment of the PDR is highlighted by the black boxes. In this patient, the PDR is of slightly higher amplitude on the left, which is somewhat atypical; in most patients, the PDR is of slightly higher amplitude on the right. Of note, if the amplitude of the PDR over the right hemisphere is more than three-fold greater than the amplitude of the PDR ove than threefold the PDR over the right hemisphere is mover the left hemisphere, it is considered abnormal. In contrast, if the amplitude of the PDR over the left hemisphere is more than two-fold greater than the amplitude of the PDR over th right hemisphere, it is considered abnormal. This applies to patients who have a dominant left hemisphere. In patients with a dominant right hemisphere, the above amplitude criteria are reversed. Settings: LFF $1 \mathrm{~Hz}, \mathrm{HFF} 70 \mathrm{~Hz}$. (B) Bipolar montage. With drowsiness, there is drop-out of the PDR and rep. With drowsing of the PDR and replacement with predominantly theta frequencies. The first vertex waves are seen in the midline, marking the onset of Stage 1 sleep (N1; red arrow). Settings: LFF $1 \mathrm{~Hz}, \mathrm{HFF} 70 \mathrm{~Hz}$. (continued)

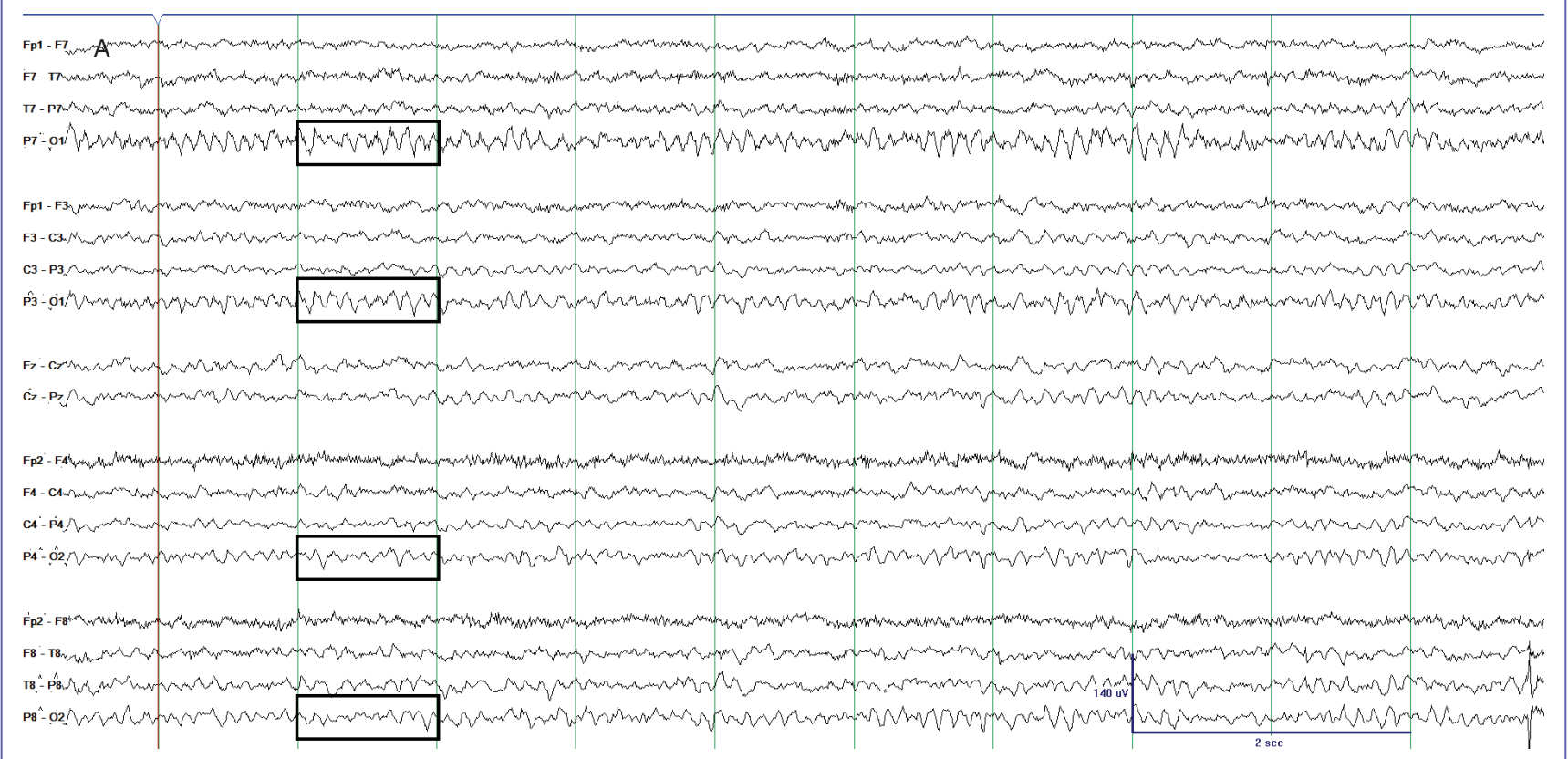

(1)




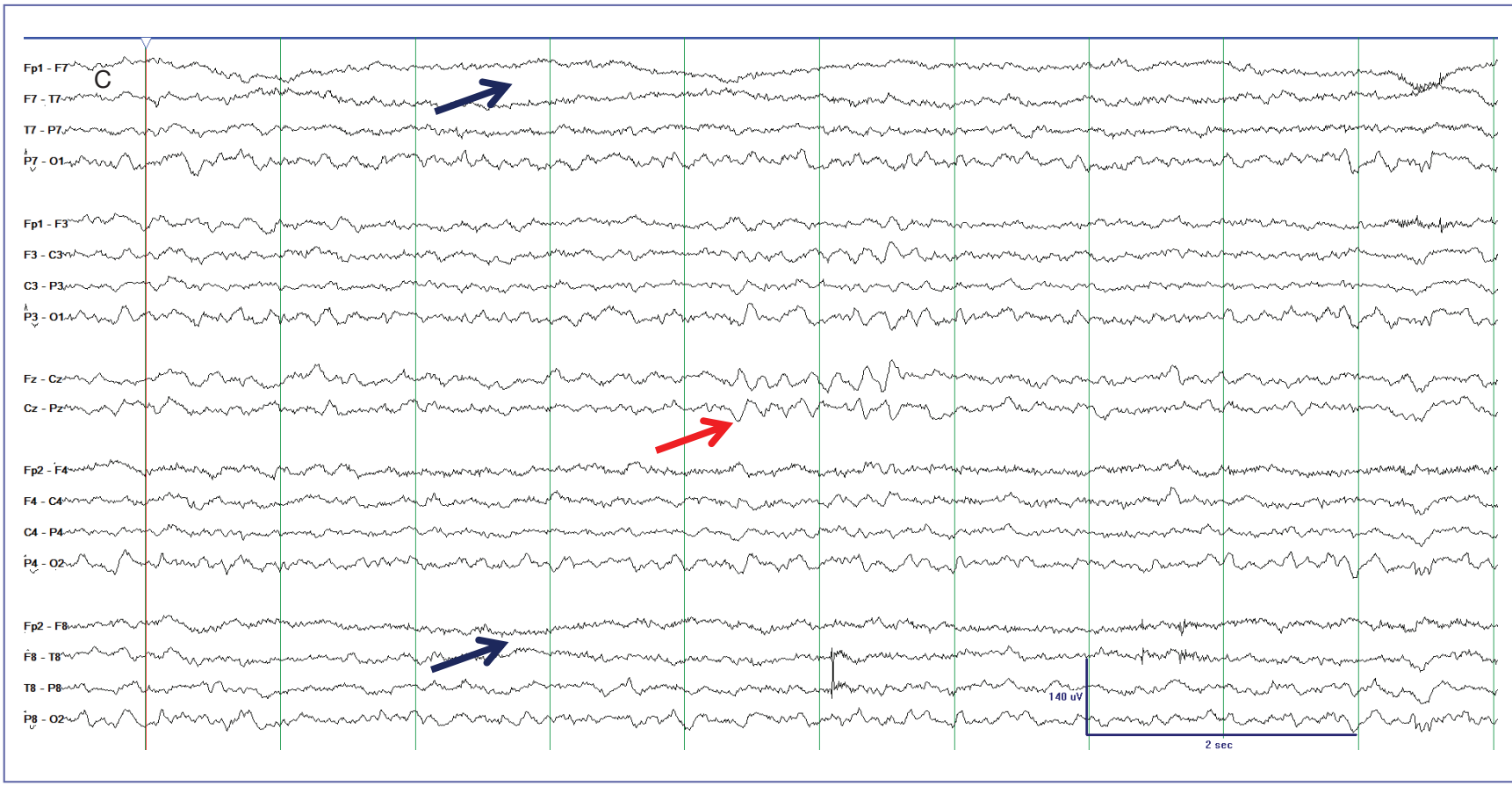

(1)
FIGURE 1.8. (continued) Normal awake and asleep EEG. (C) Bipolar montage. Vertex waves continue (red arrow), while slow roving eye movements are seen in the arrow), while slow roving eye movements are seen in the
frontal leads as slow and opposing deflections in the F7 and F8 electrodes (dark blue arrows). Of note, the eyeball is an electrical dipole with a positive charge over the cornea and a negative charge over the retina. In the case of latera eye movements, the greatest potential change is recorded over F7 and F8. With eye movement to the left, an increase in positivity is seen at F7. This manifests as an, an increase in positivity is seen at $F 7$. This manifests as an upward deflection in Fp1-F7 and a downward deflection in F7-T7. At the same time, there is an increase in negativity maximal
over F8. This manifests as a downward deflection at Fp2-F8 over F8. This manifests as a downward deflection at Fp2-F8 and an upward deflection at F8-T8. The opposite pattern is HFF $70 \mathrm{~Hz}$. (D) Bipolar montage. Onset of Stage II sleep (N2) is marked by symmetric and synchronous sleep spindles which are best seen as $14 \mathrm{~Hz}$ sinusoidal activity in the fourth second of the page (red arrow). Another spindle is seen intermixed with a vertex wave toward the end of the page
inter intermixed with a vertex wave toward the end of the page
(dark blue arrow). Settings: LFF $1 \mathrm{~Hz}, \mathrm{HFF} 70 \mathrm{~Hz}$. (continued) 


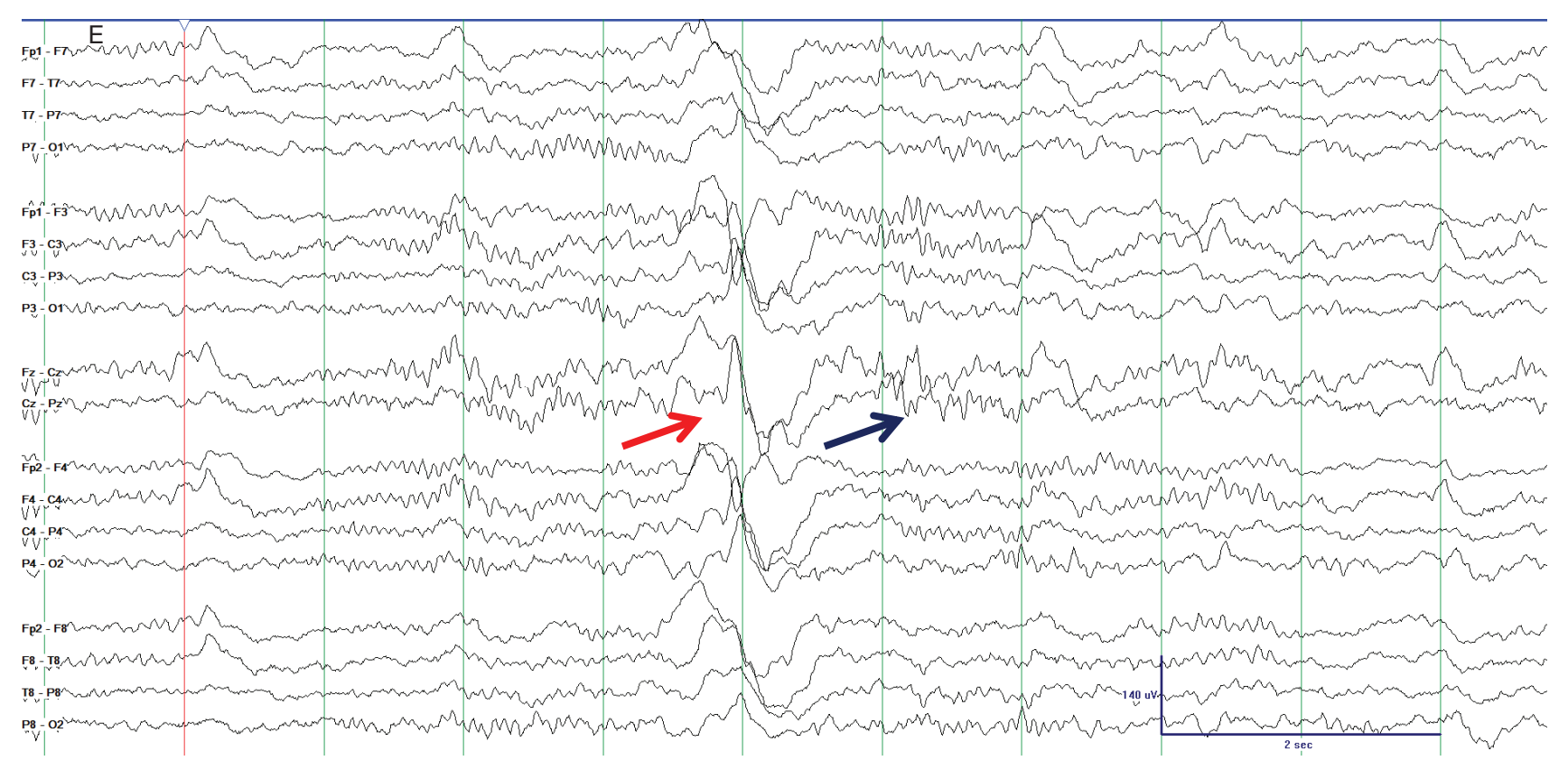

FIGURE 1.8. (continued) Normal awake and asleep EEG. (E) Bipolar montage. The large deflection in the middle of the page represents a K-complex (red arrow) followed by a sleep spindle (dark blue arrow). K-complexes are at times intermixed with a sleep spindle. Settings: LFF $1 \mathrm{~Hz}$, HFF $70 \mathrm{~Hz}$. (F) Bipolar montage. This patient progresses to deeper slow wave sleep (N3) during an overnight recording. Note the high voltage delta activity. Settings: LFF $1 \mathrm{~Hz}, \mathrm{HFF} 70 \mathrm{~Hz}$. (continued)

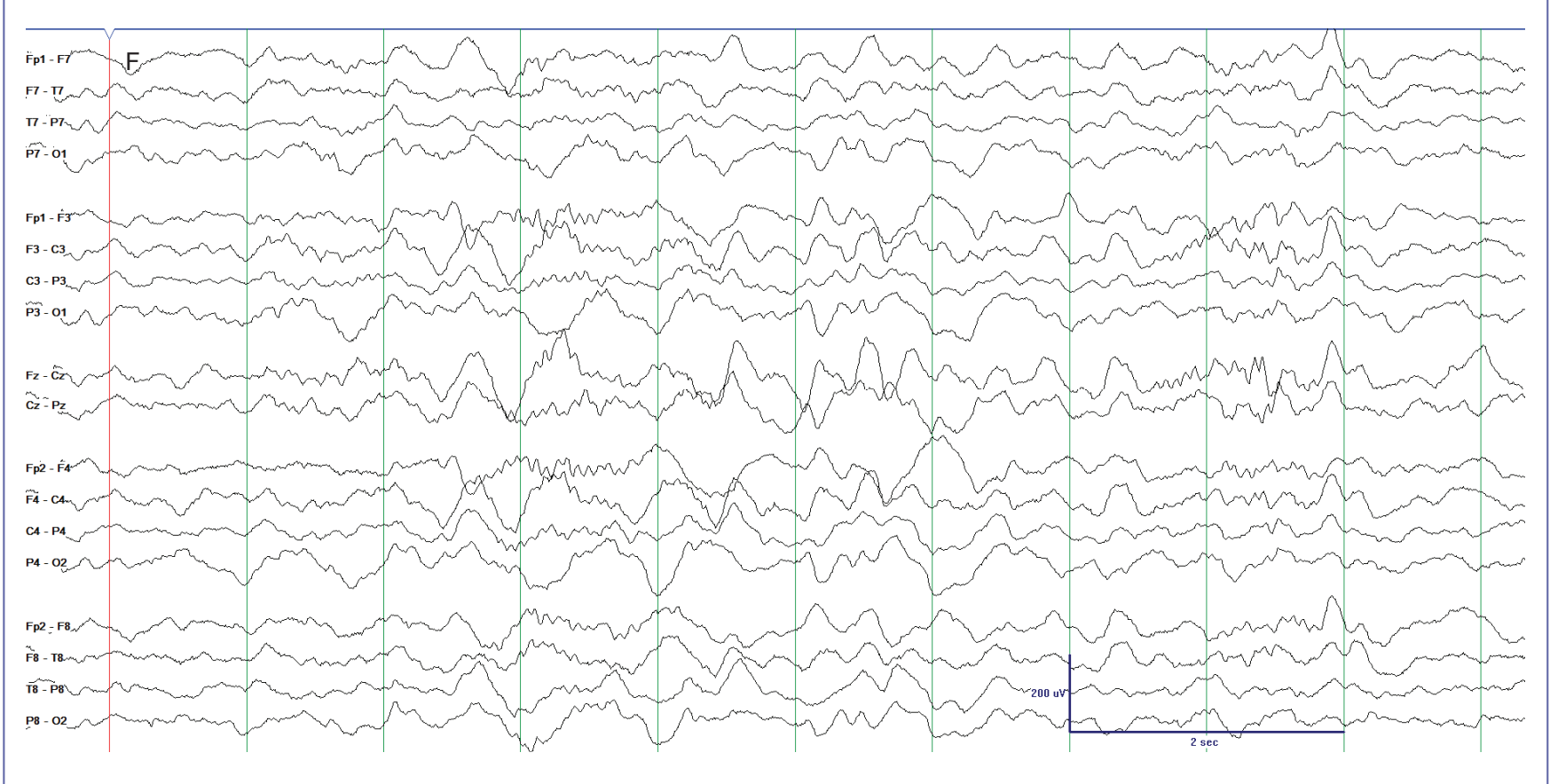



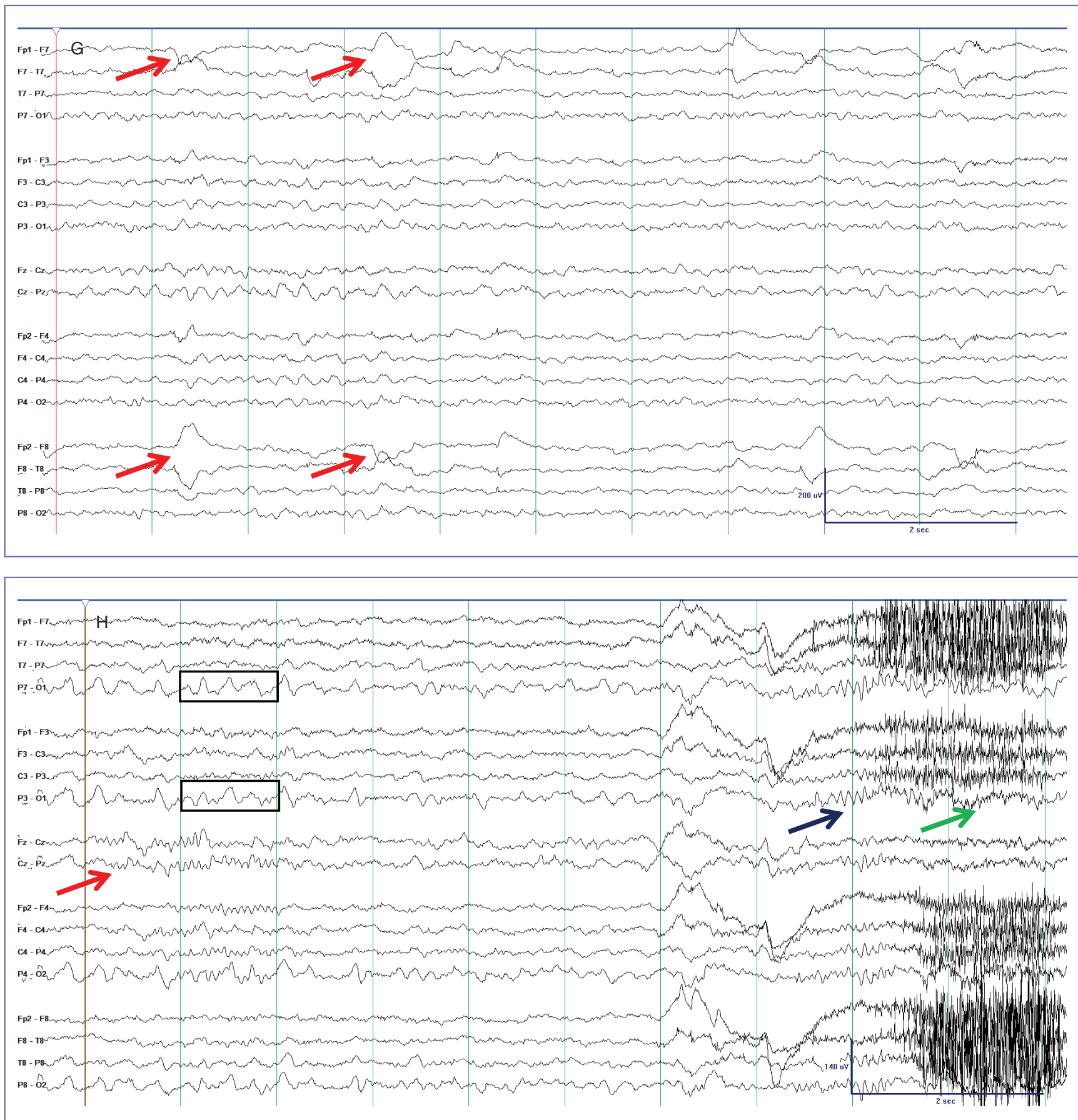

FIGURE 1.8. (continued) Normal awake and asleep EEG. (G) Bipolar montage. Later in the night, rapid eye
movement (REM) sleep is seen, with REMs in the frontal movement (REM) sleep is seen, with REMs in the fron
leads bilaterally (red arrows highlight two examples). These appear as brief, somewhat rectangular-appearing deflections in F7 and F8; compare to the slower eye movements seen in Figure 1.8C in the same location during drowsiness. Settings: LFF $1 \mathrm{~Hz}, \mathrm{HFF} 70 \mathrm{~Hz}$. (H) Bipolar montage. Still later in the night, the patient returns to and exits Stage II sleep (note spindles; red arrow) and awakens, with return of the posterior dominant rhythm (dark awakens, with return of the posterior dominant rhythm (dar
blue arrow) and muscle artifact (green arrow) related to blue arrow) and muscle artifact (green arrow) related to
movement on awakening. Note the positive occipital sharp transients of sleep (POSTS) seen over the right and left occipital regions, a subset of which are highlighted by the black boxes. POSTS are surface-positive physiologic sharp waves with a triangular or checkmark morphology seen in Stages I and II of sleep and can be asymmetric. They are a normal EEG variant, they may occur in runs at 4 to $5 \mathrm{~Hz}$, and they should not be confused with pathologic sharp waves. Settings: LFF $1 \mathrm{~Hz}, \mathrm{HFF} 70 \mathrm{~Hz}$. 
FIGURE 1.9. PDR. Bipolar montage. Normal posterior dominant rhythm in a 15-year-old awake patient. Note that the PDR is brought on by eye closure (marked by red arrow) and has a frequency of $10 \mathrm{~Hz}$. Settings: LFF $1 \mathrm{~Hz}, \mathrm{HFF} 70 \mathrm{~Hz}$.

FIGURE 1.10. Hypnagogic hypersynchrony. Bipolar montage. In infancy and early childhood, high-amplitude rhythmic theta and delta activity may be seen upon falling sleep. This is called hypnagogic hypersynchrony and is seen toward the middle of the page in this EEG. Settings: LFF $1 \mathrm{~Hz}$, HFF $70 \mathrm{~Hz}$
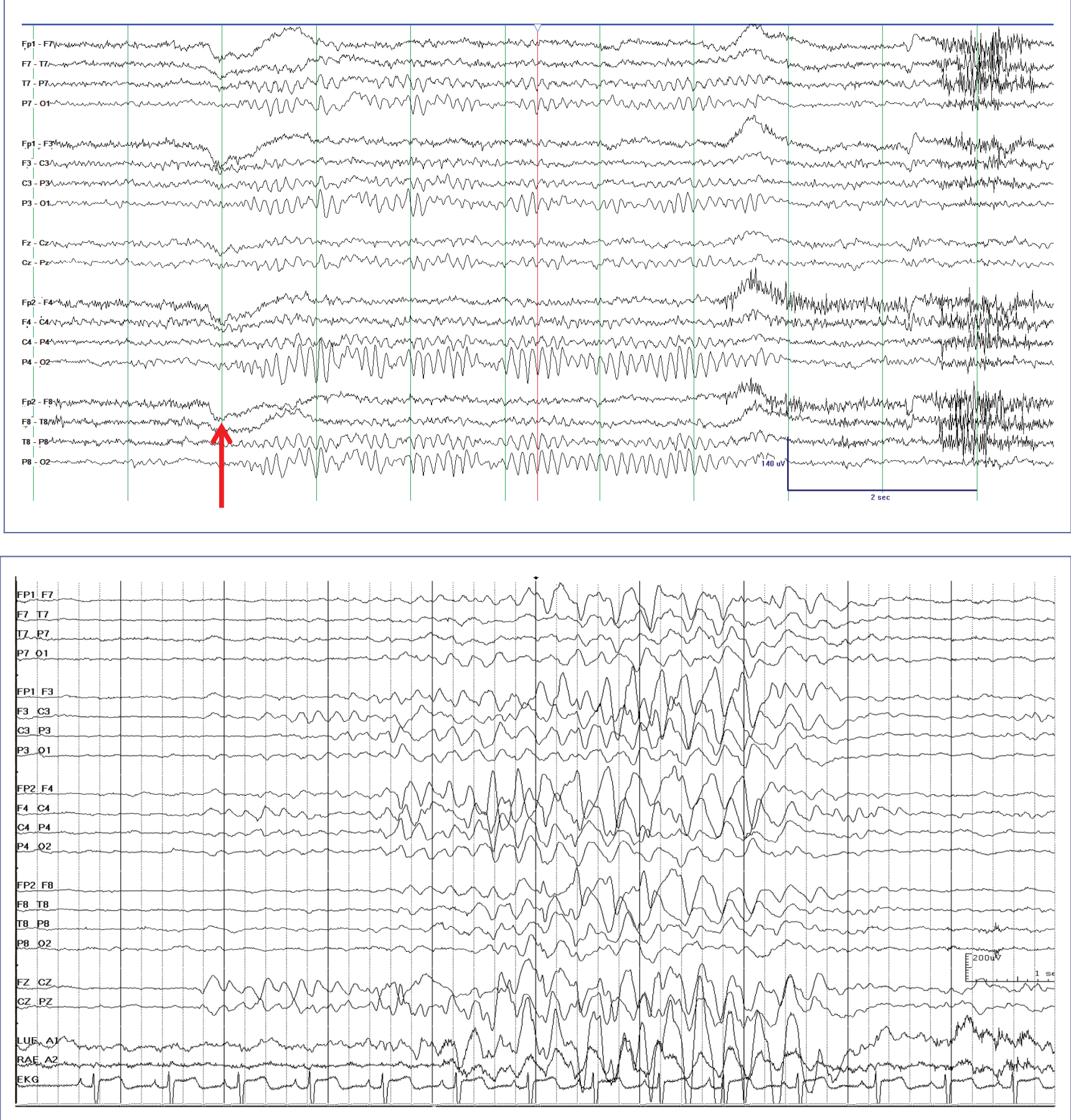


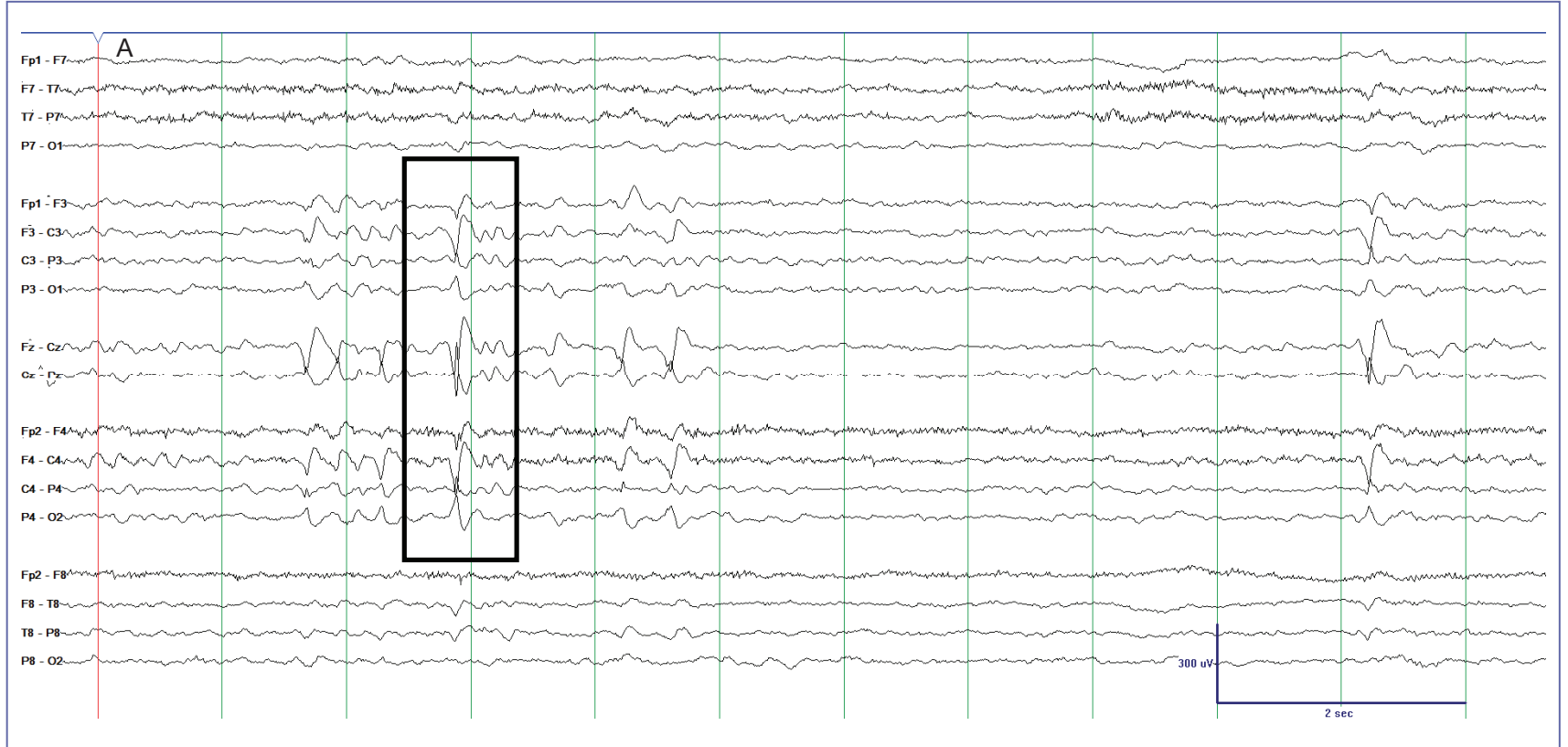

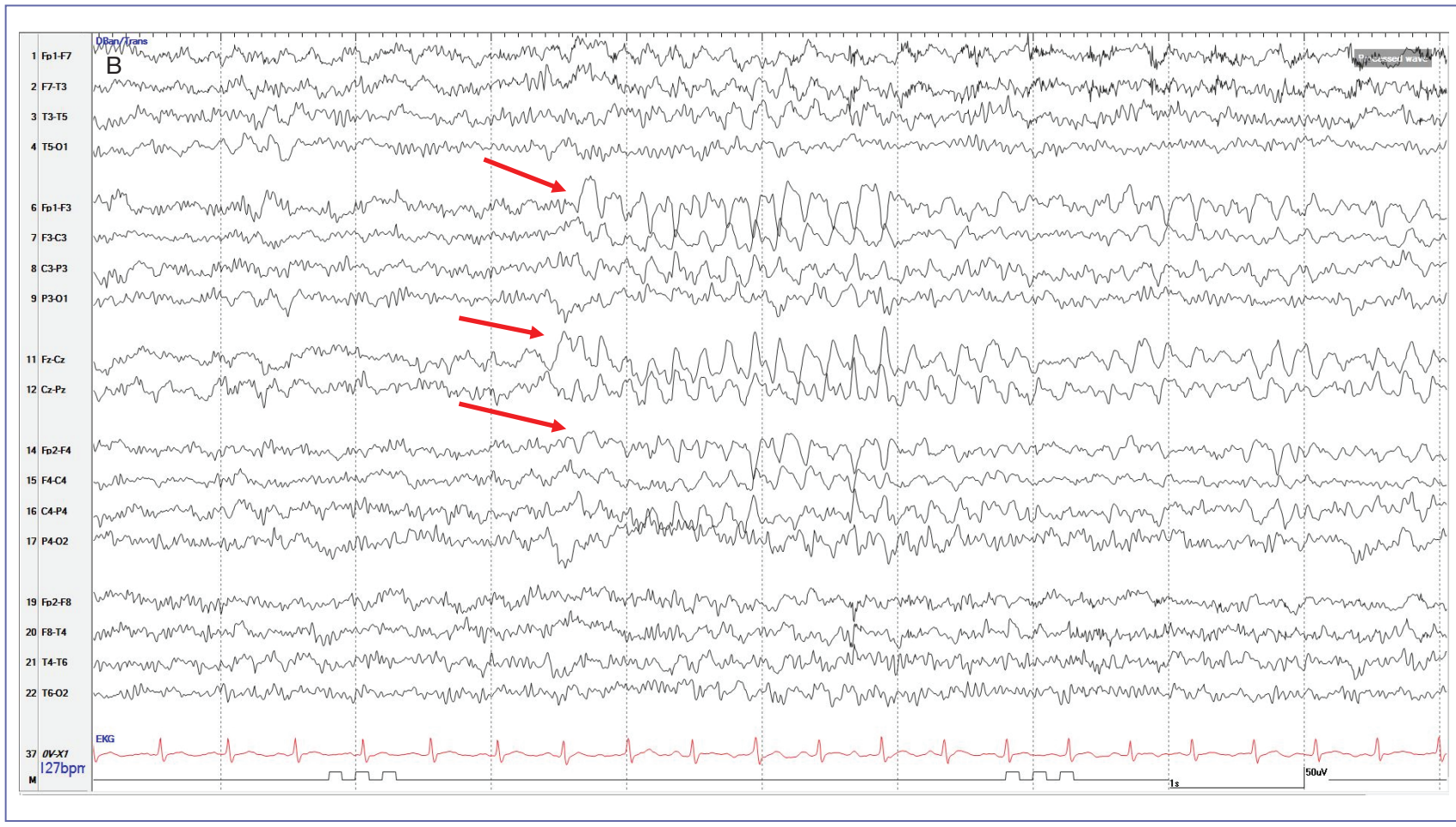

FIGURE 1.11. Sleep features. (A) Bipolar montage. Vertex waves may be spikey or very sharply contoured, as seen in this 12-year-old. This sharp contour is particularly apparent in early childhood and should not be confused with epileptiform activity. Settings: LFF $1 \mathrm{~Hz}, \mathrm{HFF} 70 \mathrm{~Hz}$. (B) Bipolar montage. Vertex waves in children may also OCcur in run 16 vertex waves. Settings: LFF $1 \mathrm{~Hz}$, HFF $70 \mathrm{~Hz}$. (continued) 


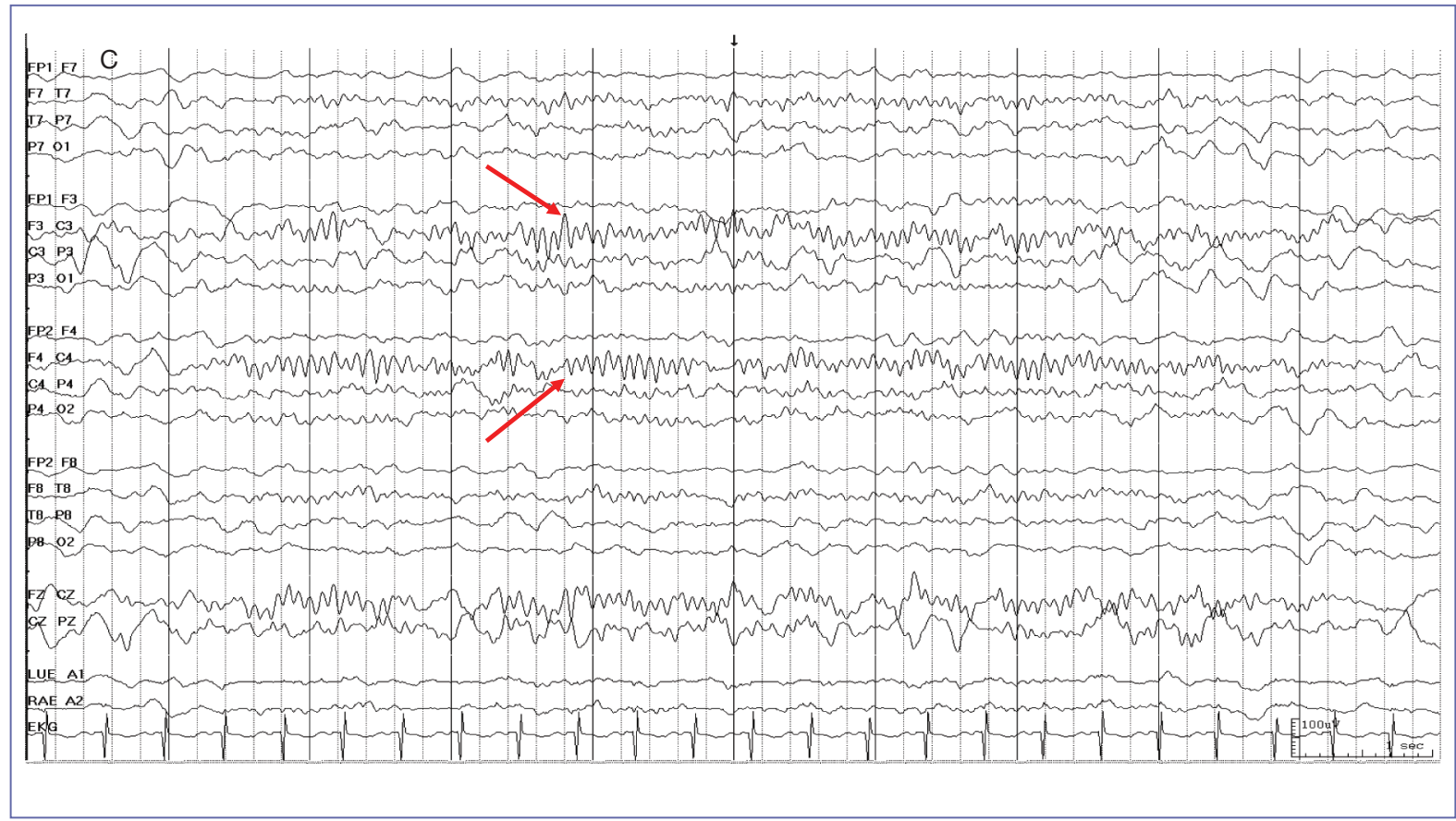

FIGURE 1.11. (continued) Sleep features. (C) Bipolar montage. Sleep spindles in infancy may be prolonged, as seen in this 4-month-old. An $\sim 8$-second long spindle is highlighted (red arrow). Spindles may last for up to 10 seconds in infants less than 6 months of age. Settings: LFF $1 \mathrm{~Hz}$, HFF $70 \mathrm{~Hz}$. (D) Bipolar montage. Sleep spindles in infancy are typically seen most prominently over the central/parietal region, and they may be een most pro here with the end of the sping 3 -month-old. The asynchrony is highighted here with the end of the spindle over the right hemisphere marked by a red arrow and the start of the spindle over the left hemisphere marked by a blue arrow. Settings: LFF $1 \mathrm{~Hz}, \mathrm{HFF} 70 \mathrm{~Hz}$.

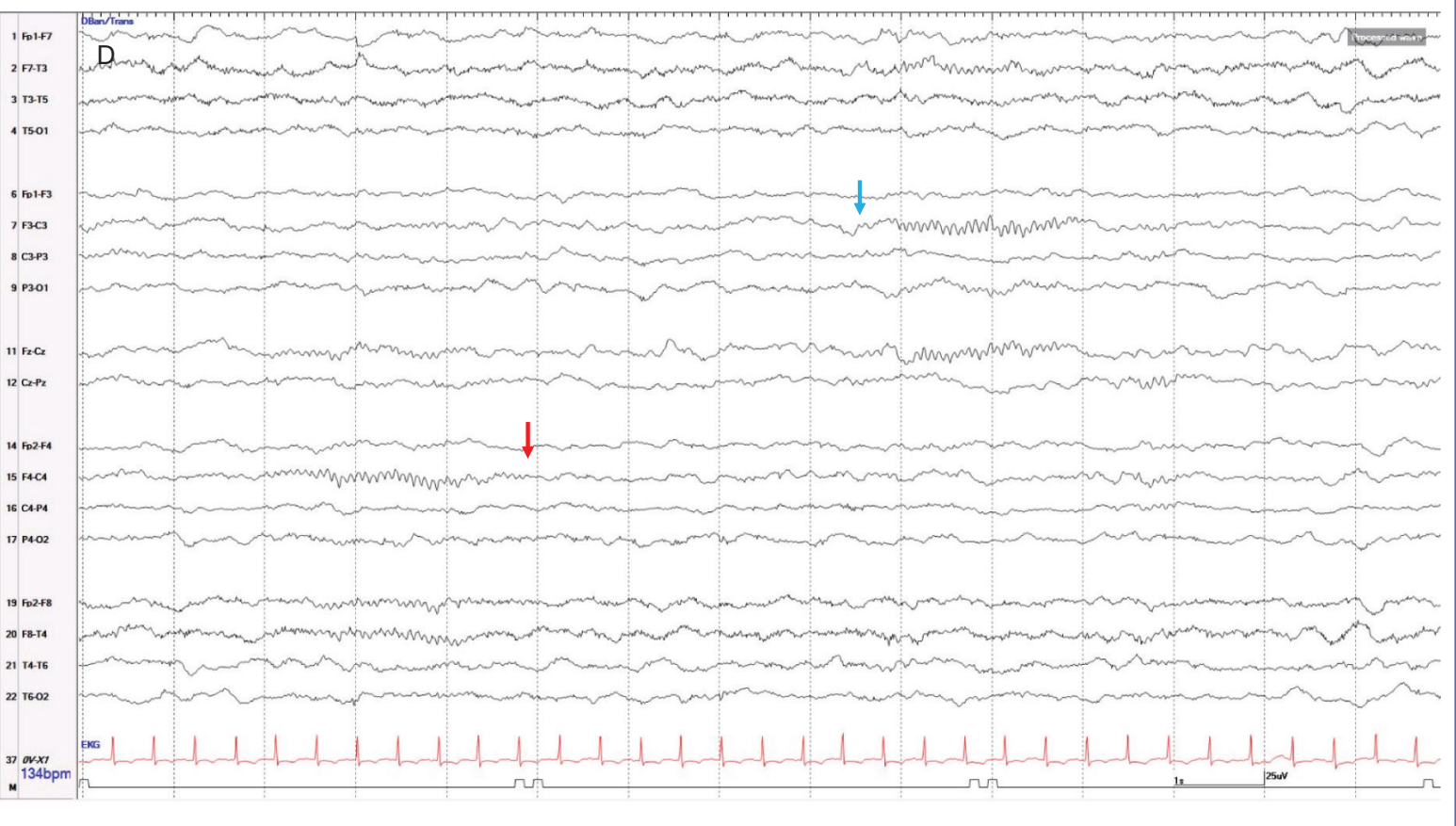



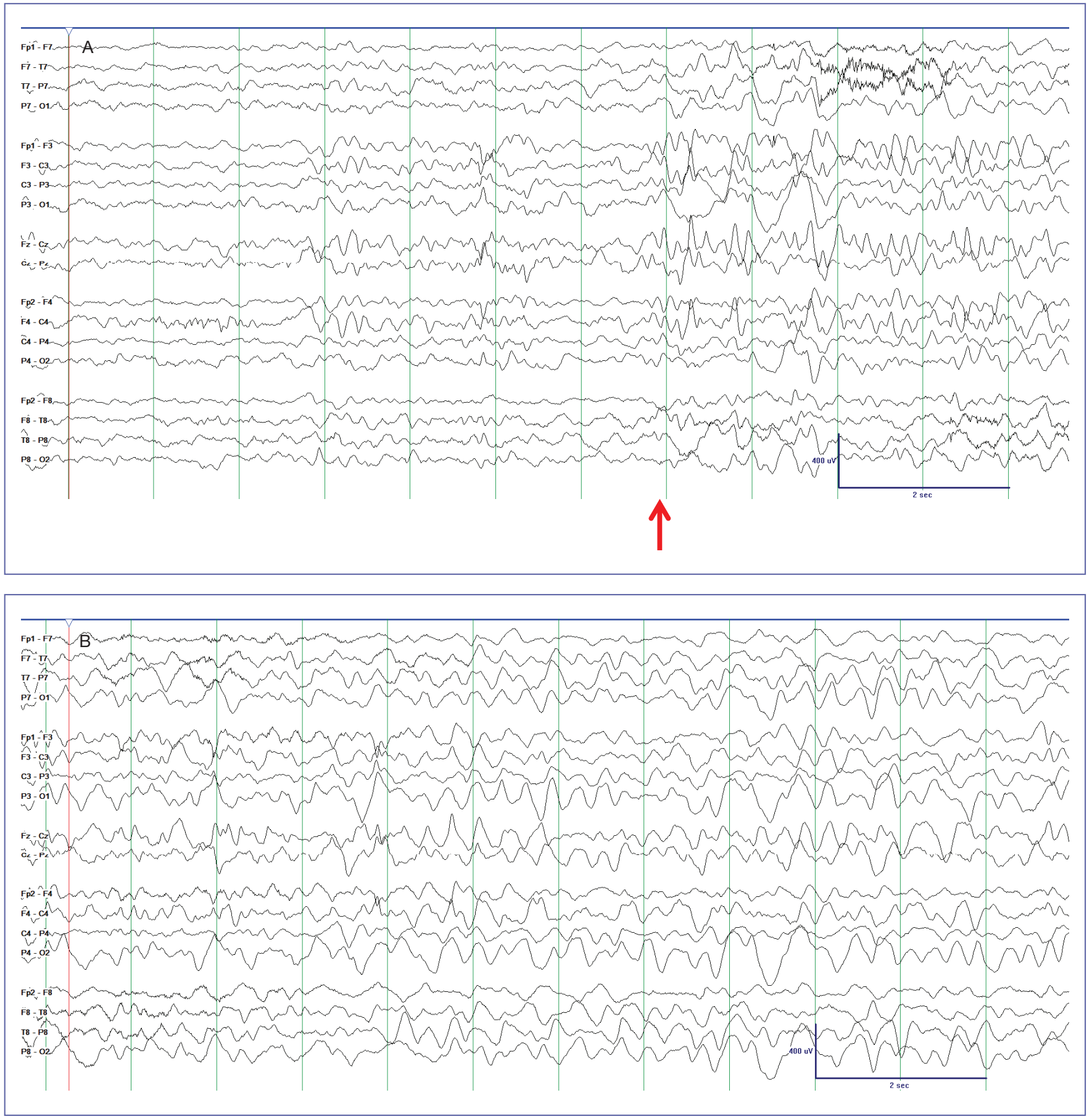

FIGURE 1.12. Hypnapompic hypersynchrony.

(A) Bipolar montage. In infancy and early childhood, highamplitude rhythmic theta and delta activity may be seen upon awakening from sleep. This is called hypnapompic hypersynchrony and is seen on the right half of the page in this 16-month-old child (onset marked by red arrow). Settings: LFF $1 \mathrm{~Hz}, \mathrm{HFF} 70 \mathrm{~Hz}$. (B) Bipolar montage. Continuation of the hypnapompic hypersynchrony that onset in Figure 1.12A. Settings: LFF $1 \mathrm{~Hz}, \mathrm{HFF} 70 \mathrm{~Hz}$. 


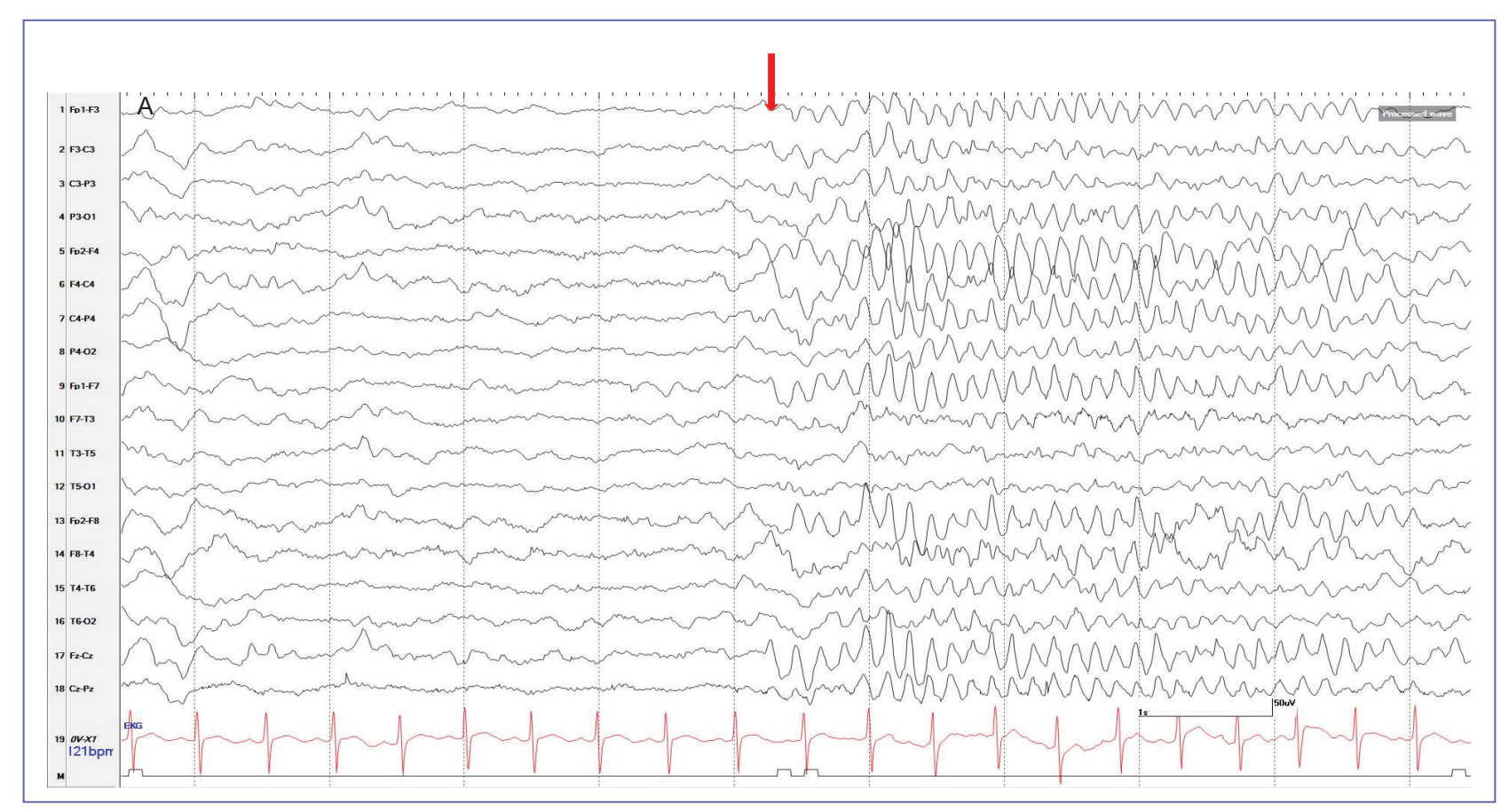

FIGURE 1.13. Arousal patterns. (A) Bipolar montage. A spontaneous arousal (red arrow) is seen in a 3-year-old with traumatic brain injury. A brief slow wave is followed by diffuse, frontally predominant theta activity. Settings: LFF $1 \mathrm{~Hz}, \mathrm{HFF}$ $70 \mathrm{~Hz}$. (B) Bipolar montage. A spontaneous arousal is seen in a 20-year-old with multifocal cerebral injury. The red arrows highlight delta slowing followed by disorganized theta and delta activity. Settings: LFF $1 \mathrm{~Hz}, \mathrm{HFF} 70 \mathrm{~Hz}$. (continued)

Source: Images courtesy of Dana Harrar.

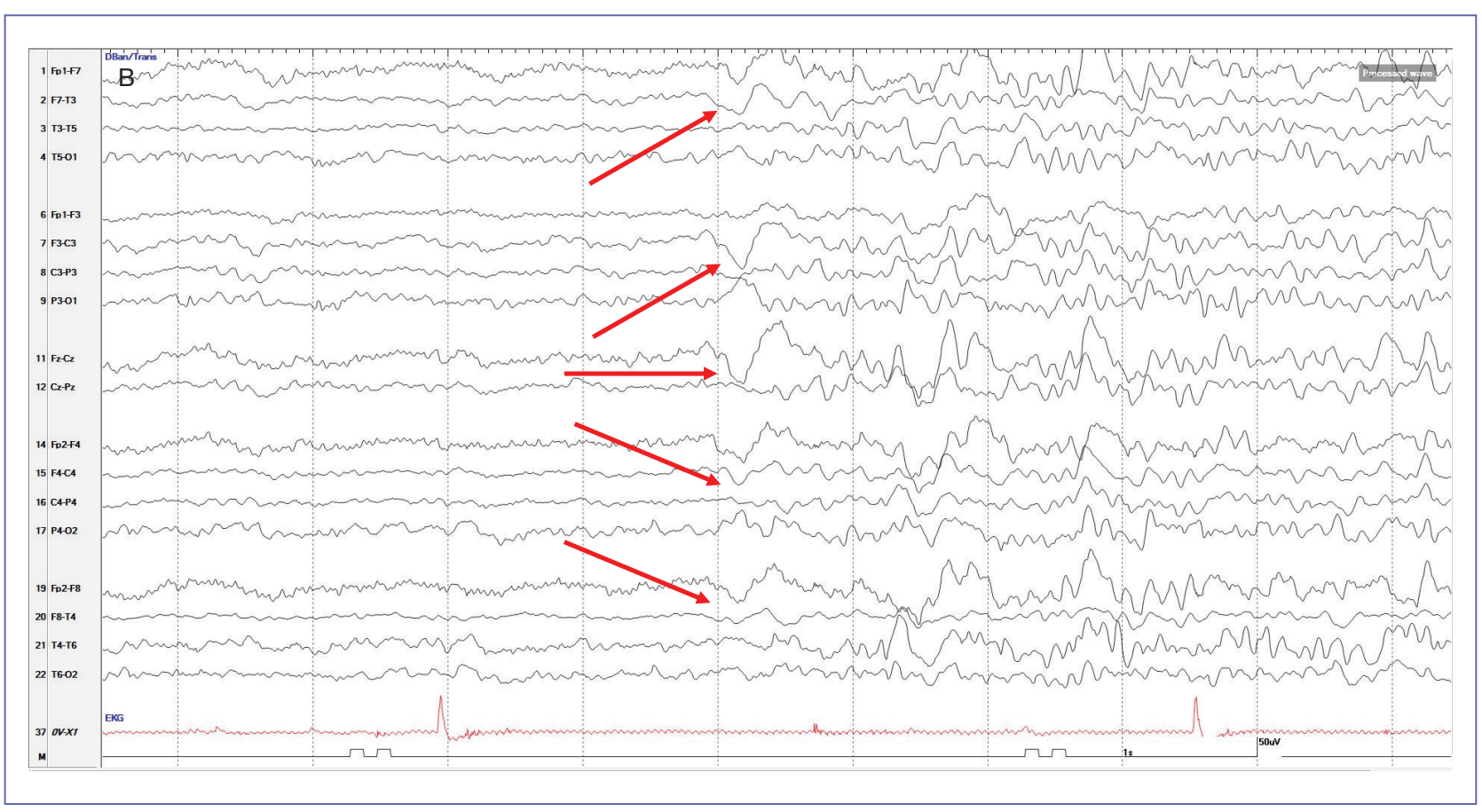



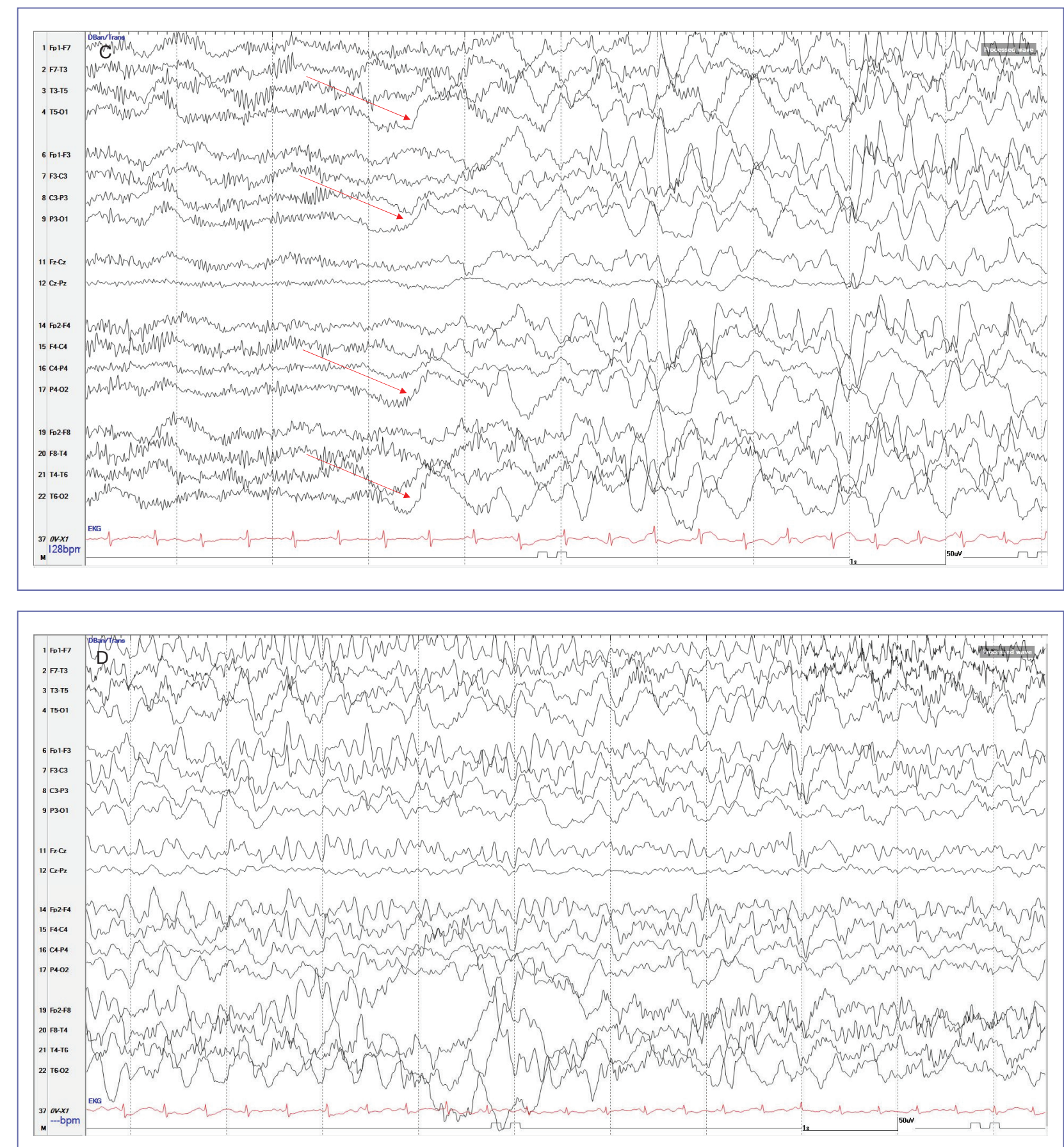

FIGURE 1.13. (continued) Arousal patterns. (C) Bipolar montage. The EEG of a 3-year-old female who presented with status epilepticus. The left side of the page is notable for diffuse beta a spontaneous arousal manifest as a posterior slow wave followed by high amplitude disorganized theta and delta activity. Settings: LFF $1 \mathrm{~Hz}$, HFF $70 \mathrm{~Hz}$. (D) Bipolar montage. Continuation of the LFF $1 \mathrm{~Hz}, \mathrm{HFF} 70 \mathrm{~Hz}$. (D) Bipolar montage. Continuation of the
arousal that onset in Figure $1.13 \mathrm{C}$, now with more prominent diffuse theta maximal over the bifrontal regions (F3, F4, Fz). Delta activity continues over the occipital regions (O1 and O2). Settings: LFF $1 \mathrm{~Hz}$, HFF $70 \mathrm{~Hz}$.

Source: Images courtesy of Dana Harrar. 
FIGURE 1.14. Slowing. Bipolar montage. EEG from a 5-year-old with hypoxic-ischemic encephalopathy. There is prominent polymorphic delta activity with admixed theta and alpha activity and overriding beta activity. There is no posterior dominant Settings: LFF $1 \mathrm{~Hz}$, HFF $70 \mathrm{~Hz}$.

FIGURE 1.15. Reactivity. Bipolar montage. EEG reactivity is documented by hand clapping, which results in the onset of generalized slow waves (red arrow) and a mild slowing of the posterior fast activity (blue arrow). Note that there is also the onset of muscle artifact in response to hand clapping (green arrow); however, muscle artifact alone does not qualify as reactivity. Settings: LFF $1 \mathrm{~Hz}, \mathrm{HFF} 70 \mathrm{~Hz}$.
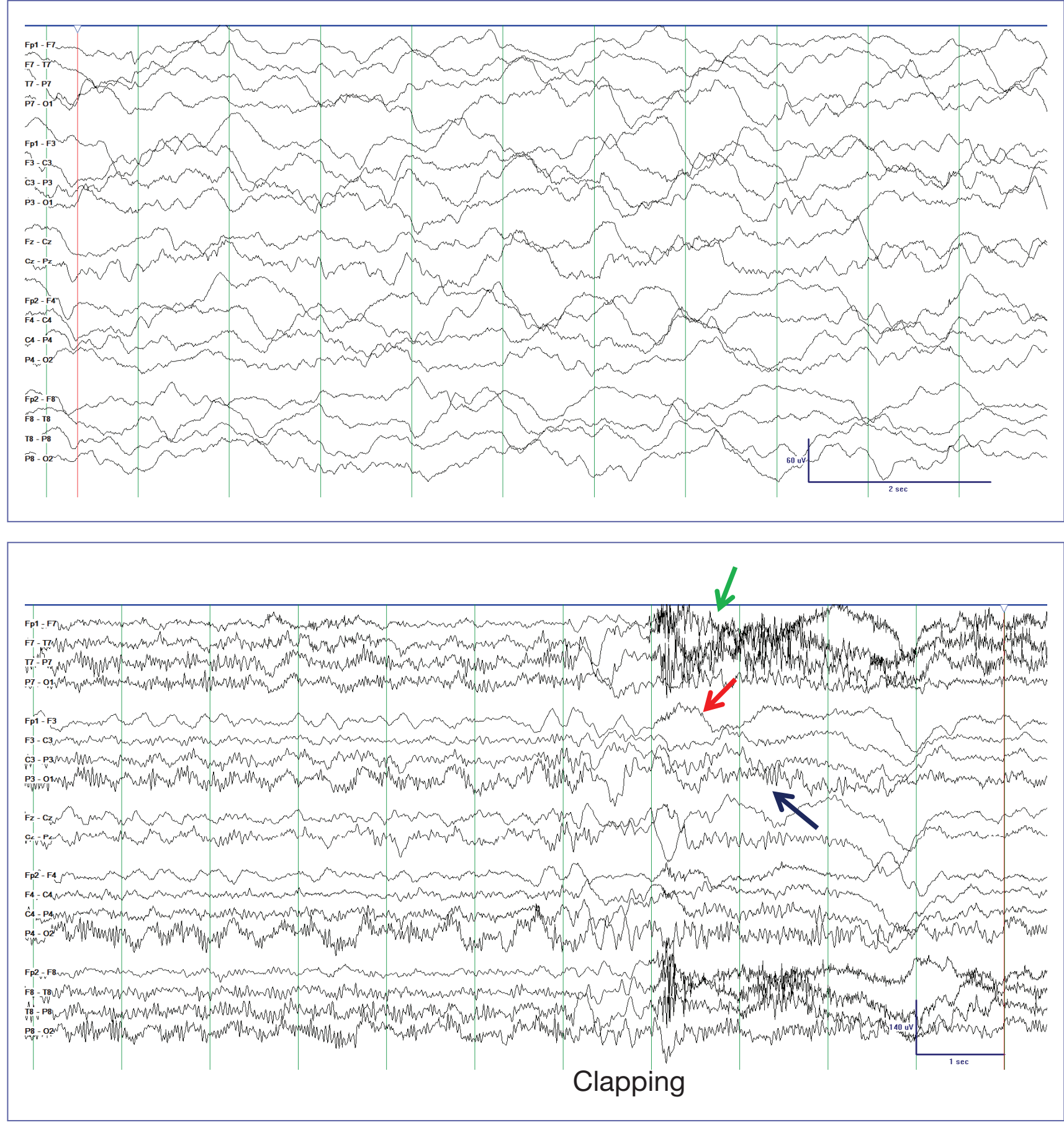
28 | Atlas of Pediatric and Neonatal ICU EEG

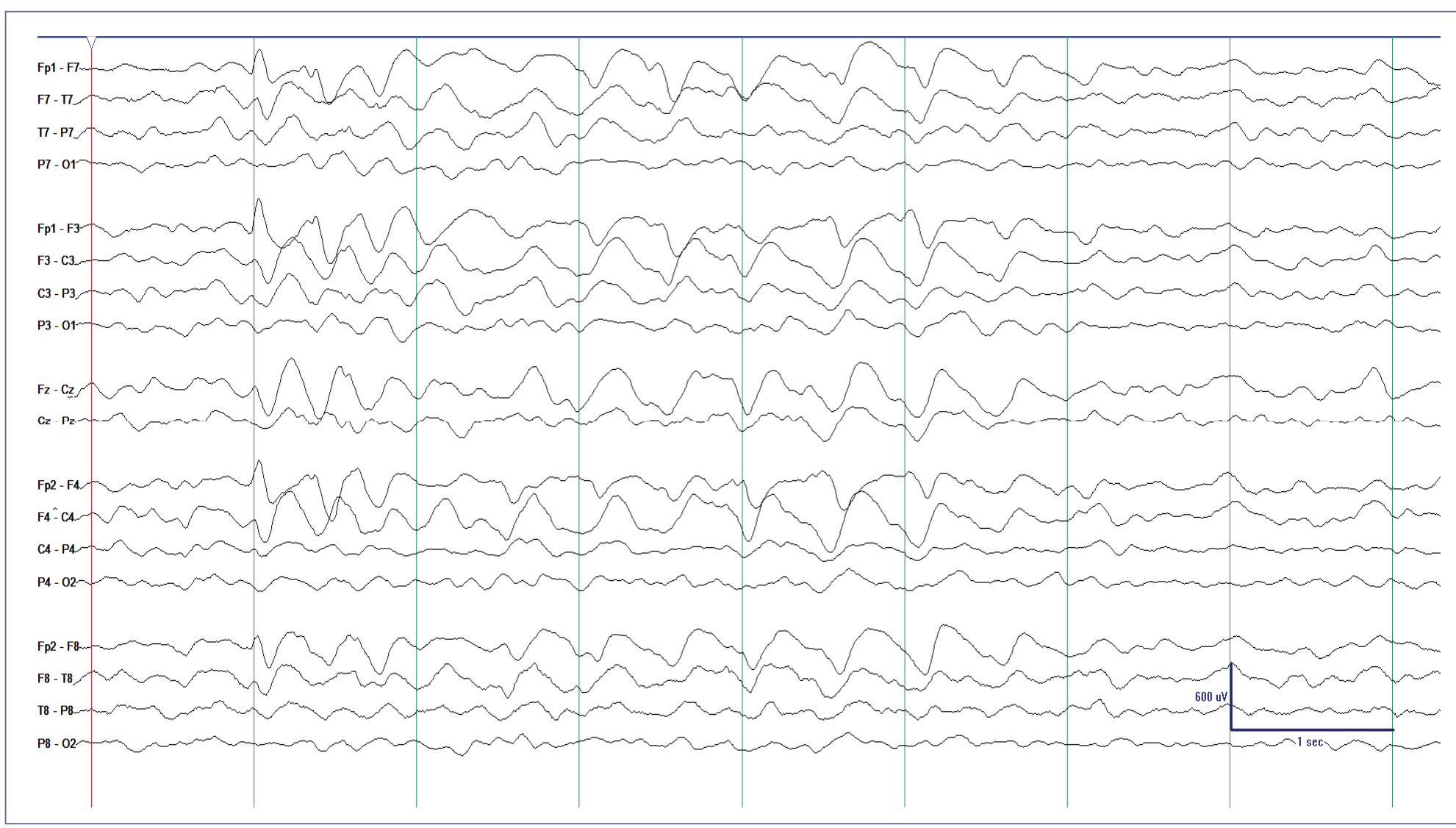

FIGURE 1.16. Generalized

rhythmic delta. Bipolar montage.

Generalized rhythmic delta activity

with bifrontal predominance in a

with bifrontal predominance in a

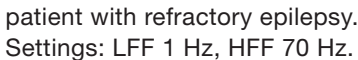




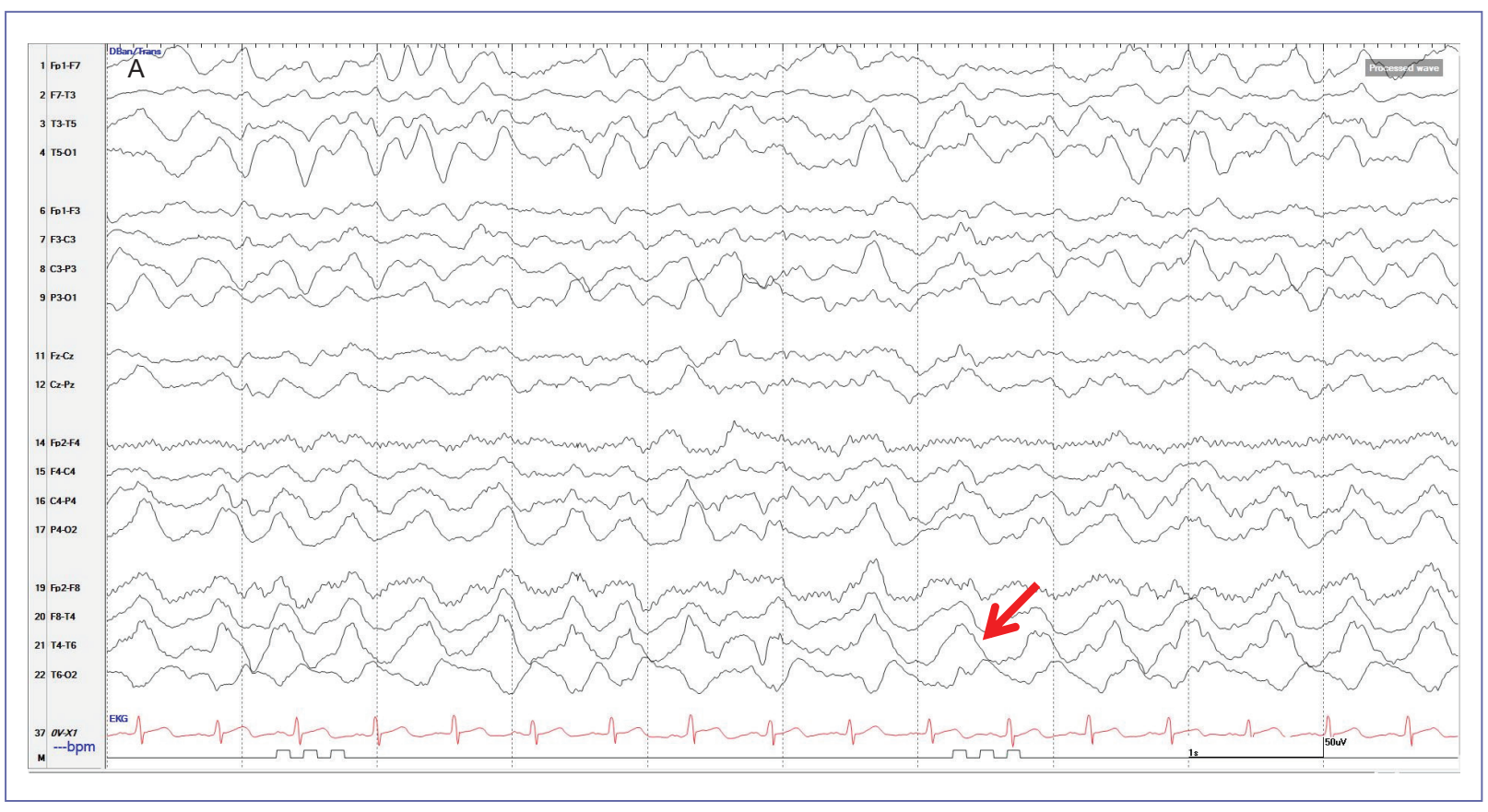

FIGURE 1.17. Lateralized rhythmic delta activity. (A) Bipolar montage. This patient with acute encephalopathy has lateralized rhythmic delta activity (RDA) over the right temporo-occipital (T6[P8]/ O2) region (red arrow). Settings: LFF $1 \mathrm{~Hz}, \mathrm{HFF} 70 \mathrm{~Hz}$. (B) Average referential montage. The same EEG as in Figure 1.17A shown on an average referential montage confirms lateralized rhythmic delta activity (RDA) maximal over T6[P8] and O2. Settings: LFF $1 \mathrm{~Hz}, \mathrm{HFF} 70 \mathrm{~Hz}$.

Source: Images courtesy of Dana Harrar.

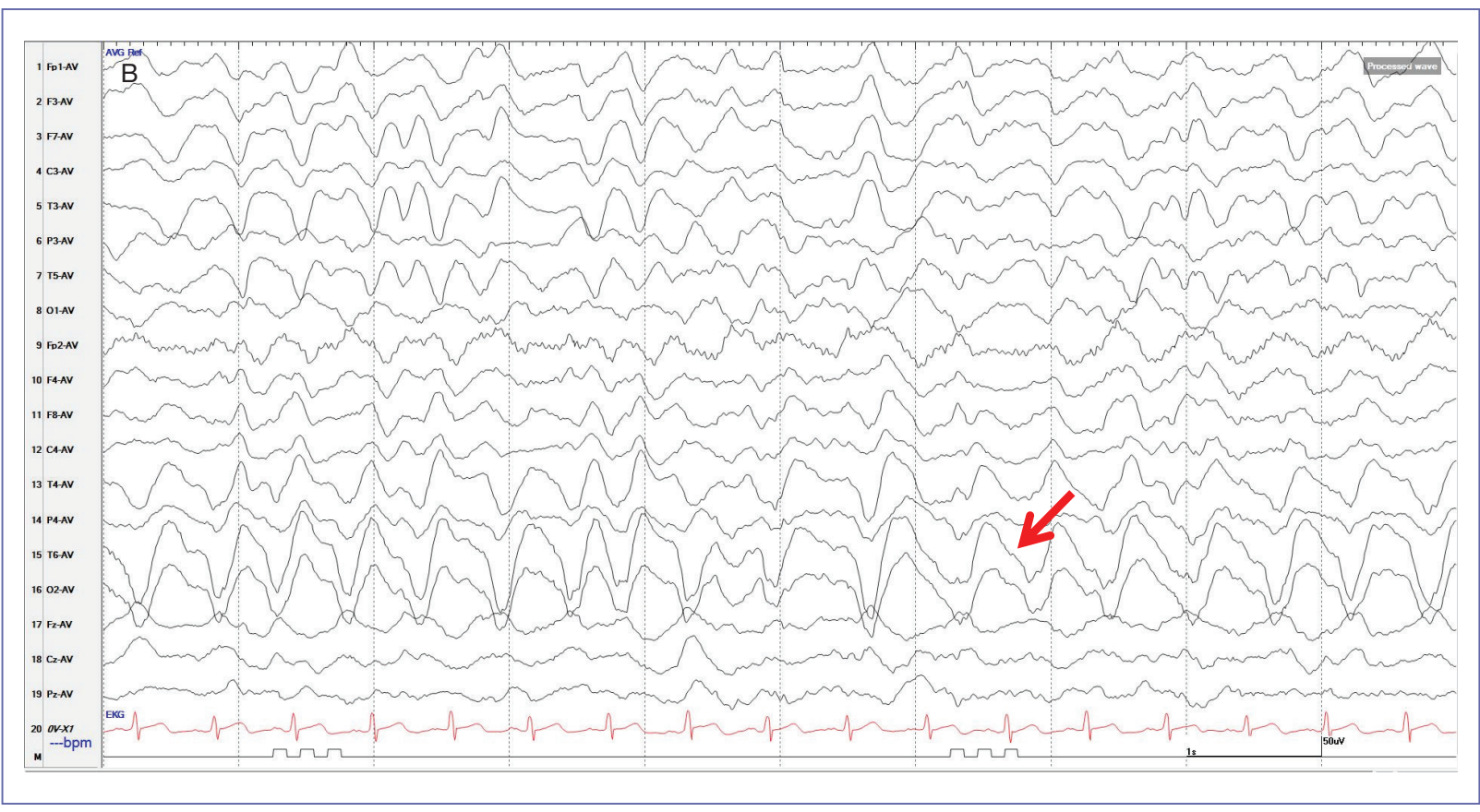



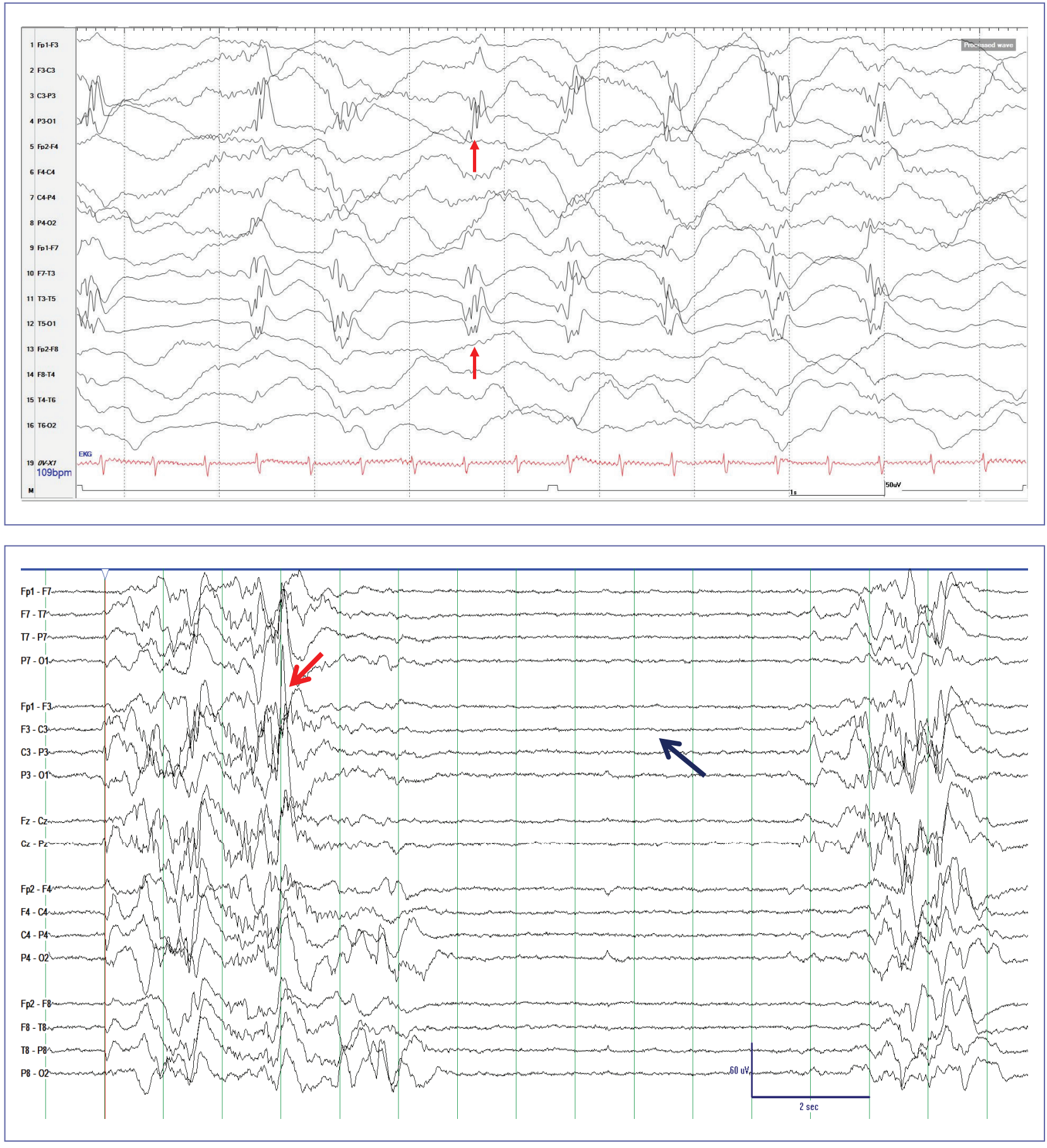

FIGURE 1.18. Periodic discharges. Bipolar montage. Lateralized periodic discharges (LPDs) over the left temporal (F7/T3[T8]/T5[P8]) and parietal (P3) regions (red arrows) in a 7-year-old with herpes simplex virus encephalitis and super-refractory status epilepticus. Settings: LFF $1 \mathrm{~Hz}, \mathrm{HFF} 70 \mathrm{~Hz}$.

FIGURE 1.19. Burst-suppression pattern. Bipolar montage. A 5 -year-old in pentobarbital coma. Note periods of suppression with voltage $<10 \mathrm{uV}$ (blue arrow) and bursts of high amplitude activity with embedded spikes (red arrow). Settings: LFF $1 \mathrm{~Hz}, \mathrm{HFF} 70 \mathrm{~Hz}$. 
FIGURE 1.20. Hemispheric asymmetry. Bipolar montage. A 9-year-old male with a progressive atypical teratoid rhabdoid tumor (ATRT). Activity ove the left hemisphere is slower and higher in amplitude than activity over the right hemisphere. Settings: LFF $1 \mathrm{~Hz}, \mathrm{HFF} 70 \mathrm{~Hz}$.

FIGURE 1.21. Asymmetric sleep features. Bipolar montage. A 2-yearold female with a right-sided subdural hematoma and a right>left watershed pattern of ischemic injury. EEG showed asymmetric sleep features. Sleep spindles are seen over the left (red arrows) but not the right hemisphere. Settings: LFF $1 \mathrm{~Hz}, \mathrm{HFF} 70 \mathrm{~Hz}$.

Source: Images courtesy of Dana Harrar.
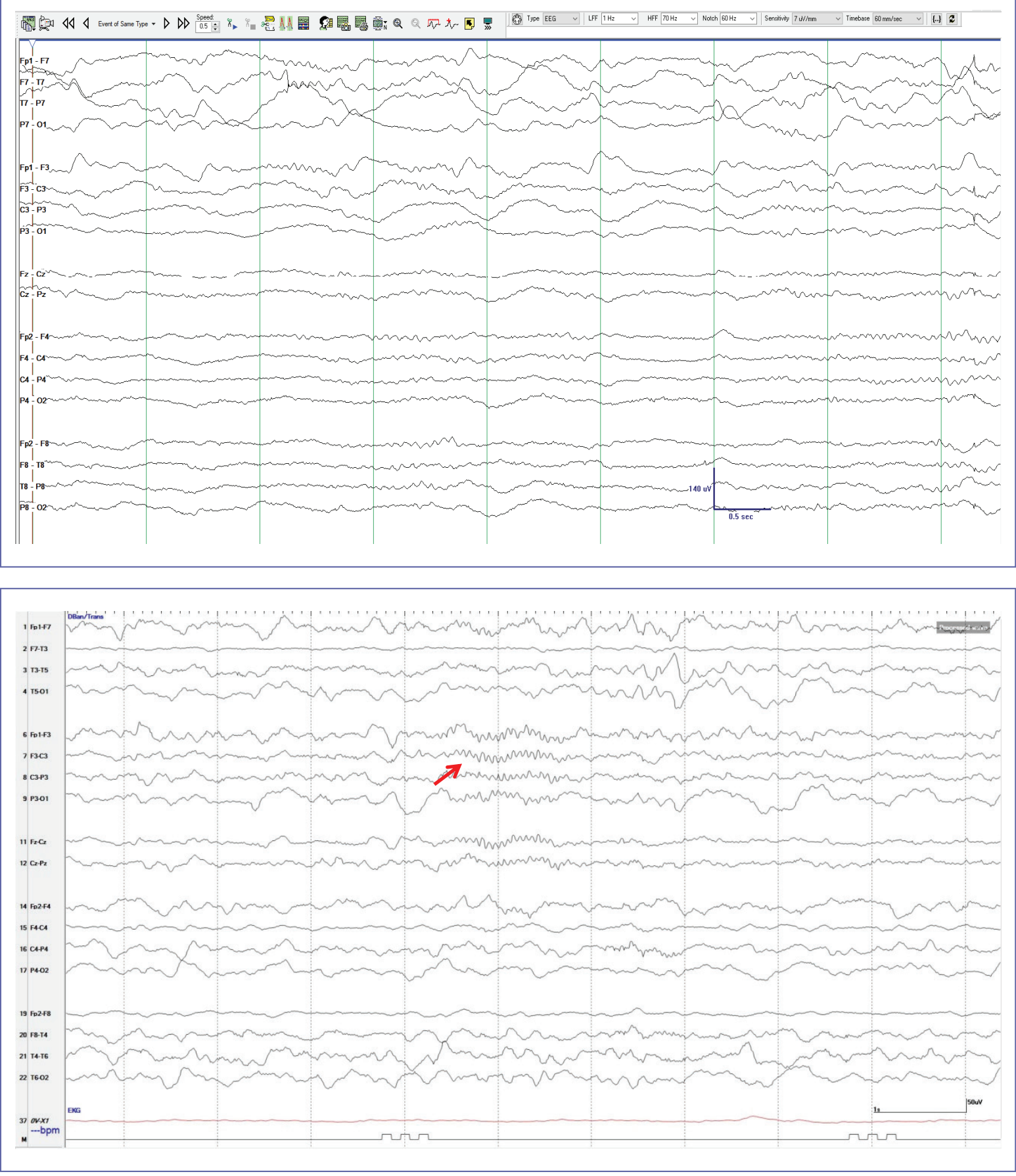


\section{References}

Only key references appear in the print edition. The full reference list appears in the digital product found on http://connect.springerpub.com/content/ book/978-0-8261-4867-4/chapter/ch01

11. Acharya JN, Hani AJ, Thirumala PD, et al. American Clinical Neurophysiology Society Guideline 3: A proposal for standard montages to be used in clinical EEG. J Clin Neurophysiol. 2016;33:312-316.

19. Berry RB, Albertario CL, Harding SM, et al. for the American Academy of Sleep Medicine. The AASM Manual for the Scoring of Sleep and Associated Events: Rules, Terminology and Technical Specifications. Darien, IL: American Academy of Sleep Medicine; 2018. Version 2.5.

20. Eeg-Olofsson O, Petersen I, Sellden U. The development of the electroencephalogram in normal children from the age of 1 through 15 years. Paroxysmal activity. Neuropadiatrie. 1971;2:375-404.
21. Petersen I, Eeg-Olofsson O. The development of the electroencephalogram in normal children from the age of 1 through 15 years. Non-paroxysmal activity. Neuropadiatrie. 1971;2:247-304.

28. Hirsch LJ, LaRoche SM, Gaspard N, et al. American Clinical Neurophysiology Society's standardized critical care EEG terminology: 2012 version. J Clin Neurophysiol. 2013;30:1-27.

30. Stecker MM, Sabau D, Sullivan L, et al. American Clinical Neurophysiology Society guideline 6: Minimum technical standards for EEG recording in suspected cerebral death. J Clin Neurophysiol. 2016;33:324-327.

31. Herman ST, Abend NS, Bleck TP, et al. Consensus statement on continuous EEG in critically ill adults and children, part II: Personnel, technical specifications, and clinical practice. J Clin Neurophysiol. 2015;32:96-108. 